\title{
CONVERGENCE AND COMPUTATION OF SIMULTANEOUS RATIONAL QUADRATURE FORMULAS*
}

\author{
U. FIDALGO PRIETO ${ }^{\dagger}$, J. R. ILLÁN ${ }^{\ddagger}$, AND G. LÓPEZ LAGOMASINO§
}

\begin{abstract}
We discuss the theoretical convergence and numerical evaluation of simultaneous interpolation quadrature formulas which are exact for rational functions. Basically, the problem consists in integrating a single function with respect to different measures by using a common set of quadrature nodes. Given a multi-index $\mathbf{n}$, the nodes of the integration rule are the zeros of the multiorthogonal Hermite-Padé polynomial with respect to $(S, \alpha, \mathbf{n})$, where $S$ is a collection of measures, and $\alpha$ is a polynomial which modifies $S$. The theory is based on the connection between Gausslike simultaneous quadrature formulas of rational type and multipoint Hermite-Padé approximation. As for the numerical treatment we present a procedure based on the technique of modifying the integrand by means of a change of variable when the integrand has real poles close to the integration interval. The output of some tests show the power of this approach when compared to other ones.
\end{abstract}

Key words. simultaneous integration rule, Gauss-like rational quadrature formula, meromorphic integrand

AMS subject classifications. Primary 41A55. Secondary 41A28, 65D32

1. Introduction. Simultaneous integration is a problem which arises in computer graphics to determinate the color of light which emanates from a given point on a surface toward the viewer (see Borges [4] and the bibliography therein). In an abstract setting, this problem was earlier studied by Nikishin [19] in connection with Hermite-Padé approximation. Simultaneous integration means that we are integrating a single function $f: I \rightarrow \mathbb{R}$, with respect to $m$ distinct measures $d s_{1}, \ldots, d s_{m}$ on $I$, respectively.

A reference index $\Phi=(O v+1) / N u$ (performance ratio) is considered in [4] to state the efficiency of the procedure. Here $O v$ is the overall degree of exactness and $N u$ is the number of integrand evaluations. Borges [4] remarked that when $m \geq 3$ the use of the $m$ corresponding Gaussian rules of polynomial type yields $\Phi<1$, which indicates a low performance. Instead he suggests quadrature rules whose nodes are the zeros of multi-orthogonal polynomials for which $\Phi>1$ holds.

Geometric rate of convergence has been proved by the authors in [8] for polynomials methods when the integrand is analytic in a neighborhood of $I$. Nevertheless, instability shows up when the corresponding numerical method is applied to meromorphic integrands with poles close to $I$. Several methods to integrate functions with singularities on or near the integration interval have been developed in the past few years.

An interesting approach, whose starting point seems to be [13], is that based on the use of rational Gaussian integration rules connected with multipoint Padé

* The work of U.F.P. and G.L.L. was partially supported by Dirección General de Enseñanza Superior under grant BFM2003-06335-C03-02 and of G.L.L. by INTAS under Grant INTAS 03-516637. The work of J.R.I. was supported by a research grant from the Ministerio de Educación y Ciencias, project code MTM 2005-01320.

${ }^{\dagger}$ Departamento de Matemáticas, Universidad Carlos III de Madrid, c/ Universidad 30, 28911 Leganés, Spain (ulises@math.uc3m.es).,

${ }_{\ddagger}^{\ddagger}$ Departamento de Matemática Aplicada I, Universidad de Vigo, Campus Universitario, 36200 Vigo, Spain (jillan@uvigo.es).

§Departamento de Matemáticas, Universidad Carlos III de Madrid, c/ Universidad 30, 28911 Leganés, Spain (lago@math.uc3m.es). 
approximation of Markov functions (cf. $[9,10,11,14,15,22])$. In what follows we will remark on some general features of the rational integration rules.

Let $\left\{\alpha_{N}\right\}_{N=1}^{\infty}$ be a sequence of algebraic polynomials with real coefficients, such that $\operatorname{deg} \alpha_{N} \leq 2 N$ and $\alpha_{N}(x)>0$, for all $N \in \mathbb{N}, x \in[a, b]$.

Let $\sigma$ be a finite and positive measure on the Borel sets of the interval $[a, b]$ and $x_{N, j}, j=1, \ldots, N$, be $N$ distinct points in $[a, b]$. Let $\Pi_{N}$ be the finite dimensional space of all polynomials of degree at most $N$. We say that the following quadrature formula

$$
\int_{a}^{b} f(x) d \sigma(x) \approx \sum_{j=1}^{N} \lambda_{N, j} f\left(x_{N, j}\right),
$$

is an interpolation formula of rational type with respect to $\alpha_{N}$ if the equality holds in (1.1) for all $f$ of the form $f=P / \alpha_{N}$, where $P \in \Pi_{N-1}$.

If $\alpha_{N} \equiv 1$ then (1.1) corresponds to the classical polynomial scheme. For every $\alpha_{N}$ it can also be considered as a the well known polynomial statement with respect to varying measures of the form $d \sigma(x) / \alpha_{N}(x)$.

Let us assume that $f$ is meromorphic in a neighborhood $V$ of $[a, b]$, and that $\{z ; f(z)=\infty\} \subset V \backslash[a, b]$. We call difficult poles to those closest to $[a, b]$ and benign to the rest. We accept such a classification of poles, though it is not rigorous at all. Under these conditions the efficiency of any numerical version of (1.1) could depend a lot on the design of polynomials $\alpha_{N}$. The presence of difficult poles in the integrand causes slow convergence of the procedure, so we have to choose a suitable strategy as that of selecting some zeros of $\alpha_{N}$ as difficult poles (cf. [9, 10, 11]).

In order to appraise in more detail the nature of this approach we consider a triangular array of complex numbers $\mathbf{T}=\left\{z_{N, j} ; j=1, \ldots, 2 N, N \in \mathbb{N}\right\}, \mathbf{T} \subset \overline{\mathbb{C}} \backslash[a, b]$, $0 \notin \mathbf{T}$, such that all its rows are symmetric with respect to the real axis. The table $\mathbf{T}$ is associated to the polynomials $\alpha_{N}$ by

$$
\alpha_{N}(x)=\prod_{j=1}^{2 N}\left(1-\frac{x}{z_{N, j}}\right) .
$$

Notice that all these polynomials $\alpha_{N}$ have real coefficients.

For the moment we assume: $z_{N, \nu} \in \mathbb{R} \backslash[a, b]$, if $\nu=1, \ldots, N$, and $z_{N, \nu}=\infty$ if $\nu=N+1, \ldots, 2 N, N \in \mathbb{N}$. In addition, $z_{N, \nu} \neq z_{N, \eta}$ for $\nu \neq \eta$. Set $P_{N-1}(x)=$ $\prod_{j \neq \nu}\left(1-x / z_{N, j}\right), \nu=1, \ldots, N$. Thus we can achieve to the value of every $\lambda_{N, j}$ by solving the following linear system

$$
\int_{a}^{b} \frac{d \sigma(x)}{\left(1-x / z_{N, \nu}\right)}=\sum_{j=1}^{N} \frac{L_{N, j}}{\left(1-x_{N, j} / z_{N, \nu}\right)}, \quad \nu=1, \ldots, N,
$$

where $L_{N, j}$ are the unknowns.

Assuming that the $N$ distinct points $x_{N, \nu}$ have been chosen according to some specific criterium, the problem (1.2) has the one and only solution $L_{N, \nu}=\lambda_{N, \nu}$, $\nu=1, \ldots, N$, formed by the so-called quadrature coefficients. An experimental fact is that the condition number of the matrix associated to the system (1.2) gets larger as $x_{N, j} / z_{N, \nu}$ get closer to one. Furthermore, numerical instability could be detected when the integral in the left side of (1.2) is calculated.

The maximum degree of exactness of formula (1.1) is $2 N-1$, and it occurs when we select as nodes the $N$ zeros of the $N$-th orthogonal polynomial with respect to the 
varying measure $d \sigma_{N}(x)=d \sigma(x) / \alpha_{N}(x)$. For such a procedure, which we are going to call rational Gauss quadrature formula (RGQF), nodes and coefficients must not be calculated from the system (1.2) but from the corresponding Jacobi matrix (cf. $[9,10,15,22])$. The numerical method to be applied is based on recursion formulas which in turn require a discretization procedure to evaluate with sufficient accuracy some integrals of the form

$$
\int_{a}^{b} \frac{P_{j}(x)}{\alpha_{N}(x)} d \sigma(x)
$$

where $P_{j}$ is a polynomial and possibly some zeros of $\alpha_{N}$ are also poles of a given integrand.

Nevertheless, when we are dealing with an $N$-point Gauss-like simultaneous formula we can not find $N$ distinct nodes in the integration interval except for some especial sets of integration measures. Moreover, these simultaneous nodes are the eigenvalues of a non-symmetric matrix and the coefficients are directly computed from a linear system of equations similar to that in (1.2).

In this paper we investigate the convergence of Gauss-like simultaneous quadrature formulas, exact for rational functions. We also present a method to evaluate three integrals of a common function by means of a rational simultaneous quadrature formula. To compute (1.3) we adopt in Section 5 a convenient change of variable as an alternative to Gautschi's algorithm when some zeros of $\alpha_{N}$ simulate difficult poles.

The paper is organized as follows.

The definition of simultaneous rational quadrature formula, the concept of ATsystem and some aspects of the simultaneous multipoint Hermite-Padé approximation, can be seen in Section 2. Likewise, in this section we apply to these formulas well known results on rational approximation to obtain convergence. These results are stated by assuming strong or weak stability of the $k$-th quadrature formula, strong normality of multi-indexes and some specific behavior of the sequence $\alpha_{N}$. The problem of obtaining this properties is discussed in the rest of the article.

In Section 3 we study the case $\mathbf{n}=(0, \ldots, 0, N, 0, \ldots, 0)$ which is reduced to the problem of evaluating two integrals simultaneously, one of them by means of a rational Gaussian rule, and the other by using an interpolation quadrature formula. In order to obtain strong stability for the latter we stablish an additional condition on the zeros of $\alpha_{N}$.

Section 4 is devoted to extend some results from the simultaneous polynomial integration to the rational version when the AT-systems have been constructed according to Nikishin's theory.

In Section 5 we present a numerical method to evaluate simultaneously three different integrals of a single function with real poles close to the integration interval. The efficiency of the method is based on the use of smoothing transformations to treat difficult poles. This section also describes correction techniques for making equal the participation of all integrators and for reducing instability due to the own recursion statement. Numerical results from two examples are compared with those obtained in earlier papers where Gaussian rules of rational type are applied.

2. Simultaneous rational quadrature formulas (SRQF). In what follows we investigate the asymptotic behavior of simultaneous quadrature formulas which are based on interpolation of rational functions. We indicate which results on convergence can be obtained for these rules when the corresponding multi-orthogonal polynomial 
of degree $N$ has exactly $N$ simple zeros in the integration interval and some (weak) stability condition holds.

We have pointed out in the previous section that rational quadrature formulas of Gauss type arose from the theory of multipoint Padé approximation of Markov's functions. A similar connection can be established between the rational approach for simultaneous approximation of integrals and multipoint Hermite-Padé approximants for Markov functions.

In the sequel a weight function $w(x)$ is a continuous function with constant sign on a given interval $[a, b]$. Let $m \in \mathbb{N}$ be fixed and $S=\left(s_{1}, \ldots, s_{m}\right)$ be a system of $m$ measures with constant sign on $[a, b]$.

From $S$ we construct $\widehat{S}=\left(\widehat{s}_{1}, \ldots, \widehat{s}_{m}\right)$, the corresponding system of Markov functions; namely,

$$
\widehat{s}_{k}(z)=\int_{a}^{b} \frac{d s_{k}(x)}{z-x}, \quad k=1, \ldots, m,
$$

In particular, we are interested in the case $d s_{k}(x)=w_{k}(x) d \mu(x)$, where $w_{k}, k=$ $1, \ldots m$, are weight functions and $\mu$ is a positive measure on $[a, b]$. We notice that all the functions $\widehat{s}_{k}$ are holomorphic in $D=\mathbb{C} \backslash[a, b]$.

Let $\mathbb{Z}_{+}^{m}=\left\{\mathbf{n}=\left(n_{1}, \ldots, n_{m}\right) ; n_{k} \in\{0,1,2, \ldots\}, k=1, \ldots, m\right\}$. For $\mathbf{n} \in \mathbb{Z}_{+}^{m}$ we put $|\mathbf{n}|=n_{1}+\cdots+n_{m}$.

For $k=1, \ldots, m$, let $\mathbf{T}_{k}$ be the table

$$
\mathbf{T}_{k}=\left\{z_{k, j} ; j \in \mathbb{N}\right\}
$$

where $\mathbf{T}_{k} \subset \overline{\mathbb{C}} \backslash[a, b], 0 \notin \mathbf{T}_{k}$. We will consider $m$ quadrature formulas of rational type, so we need $m$ polynomial sequences as those given below

$$
\alpha_{\mathbf{n}, k}(x)=\prod_{j=1}^{K}\left(1-\frac{x}{z_{k, j}}\right),
$$

where $K=K_{\mathbf{n}, k} \in \mathbb{N}$ is chosen depending on the context. For theoretical purposes we restrict to $1 \leq K \leq|\mathbf{n}|+n_{k}, k=1, \ldots m$. As for the numerical setting our method also works for $K>2|\mathbf{n}|$.

Equation (2.1) suggests that we are considering $m$ non-Newtonian triangular tables $A[1], \ldots, A[m]$ of polynomials at once, and that we are using at most the first $|\mathbf{n}|+n_{k}$ terms of the $|\mathbf{n}|$-th row of the $k$-th table to construct the polynomial $\alpha_{\mathbf{n}, k}$.

We may assume in the next sections that we are considering only one table $\mathbf{T}$; that is, $\mathbf{T}_{k}=\mathbf{T}$, and $\alpha_{\mathbf{n}}$ is the associated polynomial.

In order to obtain that all polynomials (2.1) have real coefficients, we assume that $z_{k, j} \in \mathbb{R} \cup\{\infty\} \backslash[a, b], n \in \Lambda, k=1, \ldots, m, j=1, \ldots, K$, or $\mathbf{T}$ is stable by complex conjugation.

The strategic of constructing tables $\mathbf{T}$ depends on whether the integrand has singularities in the integration interval $[a, b]$ or in $\mathbb{C} \backslash[a, b]$.

Once given a polynomial $\alpha_{\mathbf{n}, k}, k=1, \ldots, m$, one can establish the concept of simultaneous multipoint Hermite-Padé approximant $R_{\mathbf{n}}$ to $\widehat{S}$ with respect to the multiindex $\mathbf{n} \in \mathbb{Z}_{+}^{m}$ (cf. [3]). Namely, $R_{\mathbf{n}}=\left(R_{\mathbf{n}, 1}, \ldots, R_{\mathbf{n}, m}\right)$ is a vector whose components are rational functions $R_{\mathbf{n}, k}=P_{\mathbf{n}, k} / Q_{\mathbf{n}}, k=1, \ldots, m$, for which the common denominator $Q_{\mathbf{n}}$ satisfies the following properties.

$$
\operatorname{deg} Q_{\mathbf{n}} \leq|\mathbf{n}|=n_{1}+\ldots+n_{m}, \quad Q_{\mathbf{n}} \not \equiv 0
$$




$$
\frac{Q_{\mathbf{n}}(z) \hat{s}_{k}(z)-P_{\mathbf{n}, k}(z)}{\alpha_{\mathbf{n}, k}(z)}=O\left(\frac{1}{z^{n_{k}+1}}\right), \quad z \rightarrow \infty, \quad k=1,2, \ldots, m .
$$

If $Q_{\mathbf{n}}$ is the unique monic polynomial which fulfils (2.2-2.3), then it is called the Hermite-Padé polynomial associated to $(S, \mathbf{n})$, and the vector rational function $R_{\mathbf{n}}$ is the corresponding Hermite-Padé approximant (see Definition 2.1) .

The orthogonality conditions (2.4) given below, are formulated in terms of the polynomials $\alpha_{\mathbf{n}, k}$, and they can be derived from (2.3).

$$
\int_{a}^{b} t^{\nu} Q_{\mathbf{n}}(x) d s_{\mathbf{n}, k}(x)=0, \quad \nu=0, \ldots, n_{k}-1, k=1, \ldots, m,
$$

where $d s_{\mathbf{n}, k}(x)=w_{k}(x) d \mu(x) / \alpha_{\mathbf{n}, k}(x), k=1, \ldots, m$. The Hermite-Padé polynomial $Q_{\mathbf{n}}$ can also be defined as that which fulfils (2.2) and (2.4). We can also say that $Q_{\mathbf{n}}$ is the $\mathbf{n}$-th multi-orthogonal polynomial associated to the pair $\left(S,\left(\alpha_{\mathbf{n}, k}\right)_{k=1}^{m}\right), \mathbf{n} \in \mathbb{Z}_{+}^{m}$.

The basic terminology for this subject is summarized in the following definition.

Definition 2.1. A multi-index $\mathbf{n}$ is weakly normal for $\left(S,\left(\alpha_{\mathbf{n}, k}\right)\right)$ if $Q_{\mathbf{n}}$ is determined uniquely. A multi-index $n$ is said to be normal for $\left(S,\left(\alpha_{\mathbf{n}, k}\right)\right)$ if any non trivial solution $Q_{\mathbf{n}}$ of (2.4) satisfies $\operatorname{deg} Q_{\mathbf{n}}=|\mathbf{n}|$. If $Q_{\mathbf{n}}$ has exactly $|\mathbf{n}|$ simple zeros and all of them lie in the interior of $[a, b]$ the index is called strongly normal for $\left(S,\left(\alpha_{\mathbf{n}, k}\right)\right)$.

If all the indices are weakly normal, normal, or strongly normal for $\left(S,\left(\alpha_{\mathbf{n}, k}\right)\right)$, then the system $\left(S,\left(\alpha_{\mathbf{n}, k}\right)\right)$ is said to be weakly perfect, perfect or strongly perfect, respectively.

Obviously strong normality implies normality. Normality in turn, implies weak normality (see [6]).

An elementary example of strongly normal multi-index $n$ is that of the form $n_{k}=N$, and $n_{i}=0$ for $i \neq k$ (see Section 3). In the sequel, we will be interested only in subsequences $\Lambda \subset \mathbb{Z}_{+}^{m}$ of strongly normal multi-indexes with respect to $W_{k}=$ $w_{k} / \alpha_{\mathbf{n}, k}, k=1, \ldots, m$.

DEFINITION 2.2. We say that the system of functions $\left(W_{1}, \ldots, W_{m}\right)$ is an ATsystem for the multi-index $\mathbf{n}=\left(n_{1}, \ldots, n_{m}\right)$ on the interval $[a, b]$ if for every non-trivial collection of polynomials $P_{1}, \ldots, P_{m}$ with $\operatorname{deg} P_{j} \leq n_{j}-1$, the function

$$
\mathcal{H}(x)=\mathcal{H}\left(P_{1}, \ldots, P_{m}, x\right)=P_{1}(x) W_{1}(x)+\cdots+P_{m}(x) W_{m}(x),
$$

has at most $|\mathbf{n}|-1$ zeros in $[a, b]$.

The concept of AT-system, established by Nikishin in [19], is related with strong normality.

Proposition 2.3. If $\left(W_{1}, \ldots, W_{m}\right)$ is an AT-system for the multi-index $\mathbf{n}=$ $\left(n_{1}, \ldots, n_{m}\right)$ on the interval $[a, b]$ then $\mathbf{n}$ is strongly normal for $\left(S,\left(\alpha_{\mathbf{n}, k}\right)\right)$.

Propositions 2.4 and 2.5 show part of the role played by $\alpha_{\mathbf{n}, k}$ in constructing multipoint Hermite-Padé approximants.

Proposition 2.4. The following formula holds

$$
P_{\mathbf{n}, k}(z)=\int_{a}^{b} \frac{Q_{\mathbf{n}}(z) \alpha_{\mathbf{n}, k}(x)-Q_{\mathbf{n}}(x) \alpha_{\mathbf{n}, k}(z)}{z-x} d s_{\mathbf{n}, k}(x) .
$$

Proof. Define $P_{\mathbf{n}, k}$ as that in $(2.5)$ and let $k \in\{1, \ldots, m\}$. For every polynomial $q$ of degree at most $n_{k}$, the following expression for the reminder can be obtained from (2.4) and (2.5).

$$
\frac{1}{\alpha_{\mathbf{n}, k}(z)}\left(Q_{\mathbf{n}}(z) \hat{s}_{k}(z)-P_{\mathbf{n}, k}(z)\right)=\int_{a}^{b} \frac{Q_{\mathbf{n}}(x) q(x)}{q(z)(z-x)} d s_{\mathbf{n}, k}(x) .
$$


The conclusion follows from the fact that the right side of equation (2.6) satisfies (2.3).

Proposition 2.5. Assume that

$$
\operatorname{deg} \alpha_{\mathbf{n}, k} \leq|\mathbf{n}|+n_{k} \text { for } k=1,2, \ldots, m .
$$

Then $P_{\mathbf{n}, k} \in \Pi_{|\mathbf{n}|-1}$ for all $\mathbf{n} \in \mathbb{Z}_{+}^{m}$ and $k=1, \ldots, m$.

Proof. We can rewrite conveniently the expression (2.5) for $P_{\mathbf{n}, k}$ as follows

$\left(2.8 \oiint_{\mathbf{n}, k}(z)=\int_{a}^{b} \frac{\left(Q_{\mathbf{n}}(z) \alpha_{\mathbf{n}, k}(x) \pm Q_{\mathbf{n}}(x) \widetilde{\alpha}_{\mathbf{n}, k}(x) \widetilde{\widetilde{\alpha}}_{\mathbf{n}, k}(z)-Q_{\mathbf{n}}(x) \alpha_{\mathbf{n}, k}(z)\right)}{(z-x)} d s_{\mathbf{n}, k}(x)\right.$,

In the factorization $\alpha_{\mathbf{n}, k}(x)=\widetilde{\alpha}_{\mathbf{n}, k}(x) \widetilde{\widetilde{\alpha}}_{\mathbf{n}, k}(x)$, we only have assumed that $\operatorname{deg} \widetilde{\alpha}_{\mathbf{n}, k} \leq$ $n_{k}$ and $\operatorname{deg} \widetilde{\widetilde{\alpha}}_{\mathbf{n}, k} \leq|\mathbf{n}|$.

From (2.8) and the orthogonality condition (2.4) for $Q_{\mathbf{n}}$ we obtain that

$$
P_{\mathbf{n}, k}(z)=\int_{a}^{b} \widetilde{\alpha}_{\mathbf{n}, k}(x)\left(\frac{Q_{\mathbf{n}}(z) \widetilde{\widetilde{\alpha}}_{\mathbf{n}, k}(x)-Q_{\mathbf{n}}(x) \widetilde{\widetilde{\alpha}}_{\mathbf{n}, k}(z)}{z-x}\right) d s_{\mathbf{n}, k}(x) .
$$

This is all what we need to prove Proposition 2.5.

Conditions which ensure uniform convergence of Hermite-Padé approximants have been obtained in [3] when $\alpha_{N, k} \equiv 1$. The study of multipoint Padé approximation of one Markov function $(m=1)$ was initiated in [13].

Let $\mu$ be a finite, positive, Borel measure on the interval $[a, b],\left\{w_{1}, \ldots, w_{m}\right\}$ a set of weight functions, and $\mathbf{n}=\left(n_{1}, \ldots, n_{m}\right) \in \Lambda$, where $\Lambda$ is a sequence of strongly normal multi-indexes for $\left(S, \alpha_{\mathbf{n}, k}\right)$.

The general formulation of the problem of integrating simultaneously a single function under the condition that the efficiency index $\Phi$ be greater than one, leads to $m$ quadrature rules with a common set of nodes $x_{\mathbf{n}, j}, j=1, \ldots,|\mathbf{n}|$. These nodes are the $|\mathbf{n}|$ simple zeros of the $|\mathbf{n}|$-th multi-orthogonal polynomial $Q_{\mathbf{n}}$ with respect to $\left(S, \alpha_{\mathbf{n}}\right)$.

The weights $\lambda_{\mathbf{n}, k, j}$ of the $k$-th quadrature formula are given by

$$
\lambda_{\mathbf{n}, k, j}=\alpha_{\mathbf{n}, k}\left(x_{\mathbf{n}, j}\right) \int_{a}^{b} \frac{Q_{\mathbf{n}}(x)}{Q_{\mathbf{n}}^{\prime}\left(x_{\mathbf{n}, j}\right)\left(x-x_{\mathbf{n}, j}\right)} d s_{\mathbf{n}, k}(x),
$$

where $j=1, \ldots,|\mathbf{n}|, k=1, \ldots, m$. The coefficients defined by $(2.9)$ will be called Nikishin- Christoffel coefficients.

The number of integrand evaluations of these simultaneous quadratures rules is the same as in the polynomial case; that is, the parameter $N_{u}$ (see Section 1) is not affected by the presence of the polynomials $\alpha_{\mathbf{n}}$ at all.

It must be pointed out that the varying measures $d s_{\mathbf{n}, k}(x), k=1, \ldots m$, play a role in the construction of the nodes $\left(x_{\mathbf{n}, j}\right)_{j=1}^{|\mathbf{n}|}$ and the coefficients $\left(\lambda_{\mathbf{n}, k, j}\right)_{j=1}^{|\mathbf{n}|}, k=1, \ldots, m$.

The following lemma can be derived from (2.9). The technical details of the proof are the same as those in Lemma 1 of [8].

LEMMA 2.6. Let $k \in\{1, \ldots, m\}$, and let $\mathbf{n}=\left(n_{1}, \ldots, n_{m}\right)$ be a strongly normal multi-index for the system $S_{\mathbf{n}}$. Then the following equality holds

$$
\int_{a}^{b} x^{\nu} d s_{\mathbf{n}, k}(x)=\sum_{j=1}^{|\mathbf{n}|} \frac{\lambda_{\mathbf{n}, k, j}}{\alpha_{\mathbf{n}, k}\left(x_{\mathbf{n}, j}\right)} x_{\mathbf{n}, j}^{\nu}, \quad \nu=0, \ldots,|\mathbf{n}|+n_{k}-1 .
$$


Unlike the polynomial case, observe that both sides of (2.10) depend explicitely on $n$. The rational nature of (2.10) becomes more obvious if we write in the left side of the equation $\left(x^{\nu} / \alpha_{\mathbf{n}, k}(x)\right) w_{k}(x) d \mu(x)$ instead of $x^{\nu} d s_{\mathbf{n}, k}(x)$.

When $\operatorname{deg} \alpha_{\mathbf{n}, k}<|\mathbf{n}|+n_{k}-1,(2.10)$ yields a mixed interpolation procedure of polynomial-rational type.

Assume that $\Lambda \subset \mathbb{Z}_{+}^{m}$ is a sequence of strongly normal multi-indexes $n$ for $\left(S, \alpha_{\mathbf{n}}\right)$ and that $\sup _{\mathbf{n} \in \Lambda}|\mathbf{n}|=\infty$. The set of coefficients $\left\{\lambda_{\mathbf{n}, k, j}\right\}$ plays a major role in obtaining convergence properties for the corresponding integration procedures. Let $k$ be a fixed parameter $(1 \leq k \leq m)$. In what follows we discuss the role played by the weights $\left\{\lambda_{\mathbf{n}, k, j}\right\}$ in the convergence properties (cf. [8]) by assuming one or two of the following conditions.

A) For each $\mathbf{n} \in \Lambda$ all $\lambda_{\mathbf{n}, k, j}, j=1, \ldots,|\mathbf{n}|$, have the same sign as $d s_{\mathbf{n}, k}$.

B) $\sup _{\mathbf{n} \in \Lambda} \sum_{j=1}^{|\mathbf{n}|}\left|\lambda_{\mathbf{n}, k, j}\right| \leq C<\infty$.

C) $\sum_{j=1}^{|\mathbf{n}|}\left|\lambda_{\mathbf{n}, k, j}\right| \leq \kappa(n)$, where $\lim \sup _{\mathbf{n} \in \Lambda} \kappa(n)^{1 /|\mathbf{n}|}=1$.

Obviously $\mathrm{B}) \Rightarrow \mathrm{C}$ ). However, in general it is not true that $\mathrm{A}) \Rightarrow \mathrm{B}$ ), but we can modify A) slightly to obtain a condition stronger than B). Instead of A) we may state the following

A') For each $\mathbf{n} \in \Lambda$ all $\lambda_{\mathbf{n}, k, j}, j=1, \ldots,|\mathbf{n}|$, have the same sign as $d s_{\mathbf{n}, k}$, and $\operatorname{deg} \alpha_{\mathbf{n}, k} \leq|\mathbf{n}|+n_{k}-1$.

Thus we have $\left.\mathrm{A}^{\prime}\right) \Rightarrow \mathrm{B}$ ).

The problem of obtaining convergence for each one of the $m$ integration procedures must be reduced to the weaker problem of studying only the $k$-th rule. The question is: which conditions imply that

$$
\lim _{\mathbf{n} \in \Lambda,|\mathbf{n}| \rightarrow \infty} \sum_{j=1}^{|\mathbf{n}|} \lambda_{\mathbf{n}, k, j} f\left(x_{\mathbf{n}, j}\right)=\int_{a}^{b} f(x) w_{k}(x) d \sigma(x)
$$

for every $f$ in a certain non-trivial class of functions? Such a problem can be solved depending on the assumptions we adopt on $f, n, \mathbf{T}$ and on the behavior of $\sum_{j}\left|\lambda_{\mathbf{n}, k, j}\right|$ (conditions $\mathrm{A}-\mathrm{C}$ ). Moreover, the search of those values of $k$ for which convergence must be expected is a problem in itself. Assumptions A-C are very difficult to verify so a part of this paper is devoted to find sufficient conditions for them.

Definition 2.7. Let $\mathbf{T}=\left\{z_{j} ; j \in \mathbb{N}\right\}$. We say that $\mathbf{T}$ has the density property with respect to the interval $[a, b]$, if the linear space spanned by the system $\{(1-$ $\left.\left.x / z_{j}\right)^{-1} ; j \in \mathbb{N}\right\}$ is dense in $C([a, b]$ ) (complex and continuous functions on $[a, b]$ equipped with the uniform norm).

REMARKS 1.

1. It is not difficult to prove that $\mathbf{T}=\left\{z_{j} ; j \in \mathbb{N}\right\}(\mathbf{T} \subset \mathbb{C} \backslash[a, b])$ has the density property with respect to the interval $[a, b]$ if it has an accumulation point in $\mathbb{C} \backslash[a, b]$.

2. Let $\mathbf{T} \subset \mathbb{R} \backslash[-1,1]$ be formed by distinct points and let $\left\{c_{j}\right\}_{j=1}^{\infty}$ be defined as $z_{j}:=\left(c_{j}+c_{j}^{-1}\right) / 2$, with $\left|c_{j}\right|<1$. If $\sum_{j=1}^{\infty}\left(1-\left|c_{j}\right|\right)=\infty$ then $\mathbf{T}$ has the density property with respect to $[-1,1]$ (cf. [1]). 
3. Let $\mathbf{T} \subset \overline{\mathbb{C}} \backslash[0,+\infty)$ be a table such that

$$
\begin{gathered}
z_{j} \neq z_{i}, \quad \text { if } z_{j}, z_{i} \in \mathbb{C} \backslash[0,+\infty), \quad j \neq i, \\
\sum_{\Im \sqrt{z_{j}}>0} \frac{\Im \sqrt{z_{j}}}{1+\left|z_{j}\right|}=\infty .
\end{gathered}
$$

From the theory in [1] it is derived in [15] that conditions (2.12) and (2.13) yield tables for which the linear space spanned by the system $\left\{1,\left(1-x / z_{j}\right)^{-1} ; j \in\right.$ $\mathbb{N}\}$ is dense in $C([0,+\infty])$. In particular, this kind of tables have the density property (Definition 2.7) with respect to all intervals $[a, b], 0 \leq a<b \leq \infty$. An example of table $\mathbf{T}$ which satisfies (2.12) and (2.13) for $a=0, b>0$, is $z_{j}=\mathbf{i} / j, j \in \mathbb{N}$, where $\mathbf{i}$ is the imaginary unit.

The following proposition is based on well known results of the theory of rational approximation with fixed poles (cf. [23]).

Proposition 2.8. Let $S=\left(s_{1}, \ldots, s_{m}\right)$ be a system of measures on $[a, b]=[-1,1]$. For $k \in\{1, \ldots, m\}$ fixed, let $\mathbf{T}$ be a table of real numbers with $\left|z_{j}\right|>1, j=1,2, \ldots$ and $\alpha_{\mathbf{n}}$ the corresponding polynomials. Let $\Lambda \subset \mathbb{Z}_{+}^{m}$ be a sequence of distinct strongly normal multi-indexes for $\left(S, \alpha_{\mathbf{n}}\right)$. Then we have the following propositions.

1. If $\mathbf{T}$ has the density property with respect to the interval $[-1,1]$, and B) holds, then (2.11) takes place for all continuous functions $f$ on $[-1,1]$.

2. Let $d s_{k}(x)=w_{k}(x) d x$ where $w_{k}$ is a weight function on $[-1,1]$. If $\mathbf{T}$ has the density property with respect to the interval $[-1,1]$, and $\left.A^{\prime}\right)$ holds, then (2.11) takes place for all bounded Riemann integrable functions $f$ on $[-1,1]$.

3. Let $\eta=\inf _{j}\left|z_{j}\right|>1$. Also assume that C) and $\left.A^{\prime}\right)$ holds. Then (2.11) takes place for every function $f$ which is analytic for $|z|<T,(T>1)$, and real valued for $z \in(-T, T)$. Further, the following upper estimate takes place

$$
\underset{\mathbf{n}}{\limsup }\left|\int_{-1}^{1} f(x) d s_{k}(x)-\sum_{j=1}^{|\mathbf{n}|} \lambda_{\mathbf{n}, k, j} f\left(x_{\mathbf{n}, j}\right)\right|^{1 /|\mathbf{n}|} \leq \frac{\eta+T}{1+\eta T} .
$$

4. Let $f$ be a continuous function for $|z| \leq 1$ and analytic for $|z|<1$. Assume that $\left.A^{\prime}\right)$ holds and

$$
\sum_{j=1}^{\infty} \frac{\left|z_{j}\right|-1}{\left|z_{j}\right|}=\infty
$$

then (2.11) takes place.

Proof. Interpolation condition (2.10) shows that (2.11) takes place for $1 /\left(1-x / z_{i}\right)$, $i \in \mathbb{N}$. It is well known that B) and the density property of $\mathbf{T}$ with respect to $[-1,1]$ implies (2.11) for all $f \in C([-1,1])$. Hence, part (1) of Proposition 2.8 is true.

Let $f$ be a bounded and $w_{k}$-Riemann integrable function on $[-1,1]$. Without loss of generality assume that $\lambda_{\mathbf{n}, k, j} \geq 0, \mathbf{n} \in \Lambda, j=1, \ldots,|\mathbf{n}|$. From (1) it follows convergence for an arbitrary polynomial $f(x)$. In such a case the theorem of Steklov-Fejér (see Theorem 15.2.2 of [20]) asserts that (2.11) holds, so part (2) of the proposition is proved.

Let $\mathcal{E}_{\mathbf{n}, k}(f)$ be the quadrature error for the integrable function $f$, namely

$$
\mathcal{E}_{\mathbf{n}, k}(f)=\left|\int_{-1}^{1} f(x) d s_{k}(x)-\sum_{j=1}^{|\mathbf{n}|} \lambda_{\mathbf{n}, k, j} f\left(x_{\mathbf{n}, j}\right)\right| .
$$


From the interpolation condition (2.10) we obtain

$$
\left|\mathcal{E}_{\mathbf{n}, k}(f)\right| \leq\left(\int_{-1}^{1} d\left|s_{k}\right|(x)+\sum_{j=1}^{|\mathbf{n}|}\left|\lambda_{\mathbf{n}, k, j}\right|\right)\left\|f-\frac{p}{\alpha_{\mathbf{n}, k}}\right\|,
$$

where $\|\cdot\|$ is the uniform norm on $[-1,1]$ and $p \in \Pi_{|\mathbf{n}|+n_{k}-1}$.

Let $\mathbf{R}_{|\mathbf{n}|+n_{k}-1}(f)$ be the best approximation of $f$ by means of rational functions of the form $p / \alpha_{\mathbf{n}}, p \in \Pi_{|\mathbf{n}|+n_{k}-1}$, in the uniform norm on $|z|=1$, and $p_{0} / \alpha_{\mathbf{n}}, p_{0} \in$ $\Pi_{|\mathbf{n}|+n_{k}-1}$, be the best uniform approximant to $f$, on $|z|=1$. The polynomial $p_{0}$ can have complex coefficients. However, the uniqueness of the best rational approximant with fixed poles to $f$ on $|z|=1$ and the fact that $f$ and $\alpha_{\mathbf{n}}$ are real valued functions on $(-T, T)$ implies that $p_{0}$ is a polynomial with real coefficients (see [23], Chapter XII, Theorem 9). Using the maximum principle for analytic functions and taking $p=p_{0}$ in $(2.16)$ we obtain

$$
\left|\mathcal{E}_{\mathbf{n}, k}(f)\right| \leq\left(\int_{-1}^{1} d\left|s_{k}\right|(x)+\sum_{j=1}^{|\mathbf{n}|}\left|\lambda_{\mathbf{n}, k, j}\right|\right) \mathbf{R}_{|\mathbf{n}|+n_{k}-1}(f) .
$$

From [23] (Chapter IX, Theorem 6) we know that

$$
\underset{N}{\limsup } \mathbf{R}_{N}(f)^{1 / N} \leq \frac{\eta^{\prime}+T}{1+\eta^{\prime} T}, \quad\left(1<\eta^{\prime}<\eta\right)
$$

Finally, using condition C), (2.17) and the obvious inequality $\mathbf{R}_{|\mathbf{n}|+n_{k}-1}(f) \leq \mathbf{R}_{|\mathbf{n}|-1}(f)$, we obtain $(2.14)$ with arbitrary $\eta^{\prime},\left(1<\eta^{\prime}<\eta\right)$. By taking $\eta^{\prime} \rightarrow \eta$ we prove assertion (3) of Lemma 2.8 .

Part (4) is a consequence of (2.16) and ([23], Corollary 1, Ch. IX).

REMARKS 2.

1. In order to obtain convergence in part 3 of Lemma 2.8 for every function $f$ which is analytic for $|z|<T,(T>1)$, and real valued for $z \in(-T, T)$, it is sufficient that $\eta=\inf _{j}\left|z_{j}\right|>1, \alpha_{\mathbf{n}}(x)$ be given by (2.1) with $K=|\mathbf{n}|+n_{k}-1$, and

$$
\lim _{|\mathbf{n}| \rightarrow \infty}\left(\sum_{j=1}^{|\mathbf{n}|}\left|\lambda_{\mathbf{n}, k, j}\right|\right) \mathbf{R}_{|\mathbf{n}|+n_{k}-1}(f)=0 .
$$

This result follows directly from (2.17).

2. The contribution of $\alpha_{\mathbf{n}}$ in parts 1), 2) and 4) of Proposition 2.8 is relevant. Part 3) concerns with one of the most important applications of rational quadrature formulas, for which the polynomials $\alpha_{\mathbf{n}}$ play a numerical role (see [9, 10] and Section 5).

3. The connection between simultaneous rational quadrature formulas and Hermite-Padé approximants can be used to improve part 3 of Proposition 2.8. Let $f$ be analytic in a neighborhood $\Omega$ of $[-1,1]$, and $\gamma$ be a Jordan curve in $\Omega$ which surrounds $[-1,1]$. The following estimate for the quadrature error $\mathcal{E}_{\mathbf{n}, k}(f)$ is a consequence of Fubini's theorem and the Cauchy integral formula

$$
\left|\mathcal{E}_{\mathbf{n}, k}(f)\right| \leq \frac{\|f\|_{\gamma}}{2 \pi}\left\|\widehat{s}_{k}-R_{\mathbf{n}, k}\right\|_{\gamma}
$$

where $R_{\mathbf{n}, k}$ is the $k$-th multipoint Hermite-Padé approximant and $\|\cdot\|_{\gamma}$ is the supreme norm on $\gamma$. For the convergence of the right side of (2.18) see [7]. 
3. A subordination condition for a pair of weight functions. The convergence of the $k$-th rational quadrature formula of a simultaneous system depends on the asymptotic behavior of $L_{\mathbf{n}, k}=\sum_{j=1}^{|\mathbf{n}|}\left|\lambda_{\mathbf{n}, k, j}\right|$ (See estimate (2.16) and (2.17)). Here, we show how can be extended to rational quadrature formulas some convergence results based on a subordination condition imposed on a pair of weight functions. This approach was earlier considered for polynomial formulas by Sloan-Smith [21] and more recently the authors [8]. By means of this technique, we can estimate the rate of growth for $\left(L_{\mathbf{n}, k}\right)$, corresponding to a non-Gaussian quadrature formula. Let $k \in\{1, \ldots, m\}$ be fixed. The sequence of strongly normal multi-indexes $n$ to be considered is that for which the $k$-th component $n_{k}=N$ is a positive integer, and $n_{j}=0$ for $j \neq k$. Thus, the problem can be reduced to investigate simultaneous convergence for multi-indexes of the form $\mathbf{n}=(N, 0), N \in \mathbb{N}$. So in this section we always have that $|\mathbf{n}|=N$ and $m=2$.

In what follows, we additionally assume that the ordered system $S=\left(w_{1}, w_{2}\right)$ of non-negative weights fulfils the following subordination condition.

$$
C_{0}:=\left(\int_{a}^{b} \frac{w_{2}(x)^{2}}{w_{1}(x)} d \sigma(x)\right)^{1 / 2}<\infty .
$$

The common denominator $Q_{\mathbf{n}}$ is the $N$-th orthogonal polynomial associated to $w_{\mathbf{n}, 1}=$ $w_{1} / \alpha_{\mathbf{n}, 1}$. Likewise, the polynomials $P_{\mathbf{n}, k}, k=1,2$, (and consequently $R_{\mathbf{n}, k}$ ) are uniquely determined and the following expansion takes place.

$$
R_{\mathbf{n}, k}=\frac{P_{\mathbf{n}, k}(z)}{Q_{\mathbf{n}}(z)}=\sum_{j=1}^{N} \frac{\lambda_{\mathbf{n}, k, j}}{z-x_{\mathbf{n}, j}}, \quad k=1,2,
$$

where $Q_{\mathbf{n}}(z)=\prod_{j=1}^{N}\left(z-x_{\mathbf{n}, j}\right)$ and $\lambda_{\mathbf{n}, k, j}$ is given by equation (2.9). In particular, we derive from Lemma 2.6 the following formulas

$$
\int_{a}^{b} \frac{p(x)}{\alpha_{\mathbf{n}, 1}(x)} w_{1}(x) d \sigma(x)=\sum_{j=1}^{N} \lambda_{\mathbf{n}, 1, j} \frac{p\left(x_{\mathbf{n}, j}\right)}{\alpha_{\mathbf{n}, 1}\left(x_{\mathbf{n}, j}\right)}, \quad p \in \Pi_{2 N-1},
$$

and

$$
\int_{a}^{b} \frac{p(x)}{\alpha_{\mathbf{n}, 2}(x)} w_{2}(x) d \sigma(x)=\sum_{j=1}^{N} \lambda_{\mathbf{n}, 2, j} \frac{p\left(x_{\mathbf{n}, j}\right)}{\alpha_{\mathbf{n}, 2}\left(x_{\mathbf{n}, j}\right)}, \quad p \in \Pi_{N-1},
$$

where each $\alpha_{\mathbf{n}, k}(t), k=1,2$, is defined according to (2.1) with $K_{\mathbf{n}, 1}=2 N$ and $K_{\mathbf{n}, 2}=N$ respectively.

Assume that $\mathbf{T}_{k}$ is the table which corresponds to $\alpha_{\mathbf{n}, k}(t), k=1,2$, and that the following condition holds: there exist two positive constants $C_{1}, C_{2}$, such that

$$
C_{1} \alpha_{\mathbf{n}, 2}(t)^{2} \leq \alpha_{\mathbf{n}, 1}(t) \leq C_{2} \alpha_{\mathbf{n}, 2}(t)^{2}, \quad t \in[a, b], N \in \mathbb{N} .
$$

REMARKS 3. The relation $\alpha_{\mathbf{n}, 2}(t)^{2}=\lambda \alpha_{\mathbf{n}, 1}(t), \lambda>0$, trivially implies (3.5) but it is very restrictive. Other examples of polynomials satisfying (3.5), both having simple zeros, can also be constructed. Let $z_{1, j}=1 / A_{j}, z_{2, j}=1 / B_{j}, j \in \mathbb{N}$ be such that $A_{j}, B_{j} \in(0, \varepsilon)$, where $0<\varepsilon<1$, and $B_{j} \geq \max \left\{A_{2 j}, A_{2 j-1}\right\}, j \in \mathbb{N}$. In addition assume that $B_{j}=A_{2 j}+s_{j}, B_{j}=A_{2 j-1}+s_{j}^{\prime}, j \in \mathbb{N}$, where $\sum_{j=1}^{\infty}\left|s_{j}\right|<\infty$, 
$\sum_{j=1}^{\infty}\left|s_{j}^{\prime}\right|<\infty$. If $\left(z_{1, j}\right)$ and $\left(z_{2, j}\right)$ are associated to $\alpha_{\mathbf{n}, 1}$ and $\alpha_{\mathbf{n}, 2}$ respectively, then (3.5) holds for $t \in[-1,1]$ and the constants $C_{1}$ and $C_{2}$ are given by

$$
C_{1}=\prod_{j=1}^{\infty} \frac{1+A_{j}}{\left(1+B_{j}\right)^{2}}, \quad C_{2}=\prod_{j=1}^{\infty} \frac{1-A_{j}}{\left(1-B_{j}\right)^{2}} .
$$

We also remark that, given $\mathbf{T}_{2}$, the above procedure shows how $\mathbf{T}_{1}$ can be constructed so that (3.5) holds.

Theorem 3.1. Let $\Lambda$ be the sequence of multi-indexes $\mathbf{n}=(N, 0), N \in \mathbb{N}$. Let $\alpha_{\mathbf{n}, k}, k=1,2$, be polynomials which satisfy (3.5). Then

$$
\sum_{j=1}^{N}\left|\lambda_{\mathbf{n}, 2, j}\right|=\mathcal{O}\left(\left[\sum_{j=1}^{N} \lambda_{\mathbf{n}, 1, j}\right]^{1 / 2}\right)
$$

Proof. All multi-indexes in $\Lambda$ are strongly normal, because $Q_{\mathbf{n}}$ is the $N$-th orthogonal polynomial with respect to the measure $d \mu_{\mathbf{n}, 1}=\left(w_{1} / \alpha_{\mathbf{n}, 1}\right) d \sigma$ on $[a, b]$.

Condition (3.1) can be interpreted as $U(x)=w_{2}(x) / w_{1}(x) \in L^{2}\left(w_{1} d \sigma\right)$ (cf. [21]). Let $\left(Q_{\mathbf{n}, 1, \nu}\right)_{\nu=1}^{\infty}$ be the sequence of orthogonal polynomials associated to the measure $d \mu_{\mathbf{n}, 1}(x)$, normalized as $\left\|Q_{\mathbf{n}, 1, \nu}\right\|=1$. In particular $Q_{\mathbf{n}, 1, N}=Q_{\mathbf{n}}$.

Let $S_{\mathbf{n}, i}(x)$ be the $i$-th partial sum of the Fourier series of $U(x) \alpha_{\mathbf{n}, 1}(x) / \alpha_{\mathbf{n}, 2}(x)$. Taking into account that $\operatorname{deg} Q_{\mathbf{n}}(x) /\left(x-x_{\mathbf{n}, j}\right)=N-1$ and (3.3) we can deduce

$$
\begin{gathered}
\lambda_{\mathbf{n}, 2, j}=\frac{\alpha_{\mathbf{n}, 2}\left(x_{\mathbf{n}, j}\right)}{\alpha_{\mathbf{n}, 1}\left(x_{\mathbf{n}, j}\right)} \int_{a}^{b} \frac{Q_{\mathbf{n}}(x) U(x) \alpha_{\mathbf{n}, 1}\left(x_{\mathbf{n}, j}\right) w_{1}(x)}{Q_{\mathbf{n}}^{\prime}\left(x_{\mathbf{n}, j}\right)\left(x-x_{\mathbf{n}, j}\right) \alpha_{\mathbf{n}, 2}(x)} d \sigma(x)= \\
\frac{\alpha_{\mathbf{n}, 2}\left(x_{\mathbf{n}, j}\right)}{\alpha_{\mathbf{n}, 1}\left(x_{\mathbf{n}, j}\right)} \int_{a}^{b} \frac{Q_{\mathbf{n}}(x) S_{\mathbf{n}, N-1}(x) \alpha_{\mathbf{n}, 1}\left(x_{\mathbf{n}, j}\right) w_{1}(x)}{Q_{\mathbf{n}}^{\prime}\left(x_{\mathbf{n}, j}\right)\left(x-x_{\mathbf{n}, j}\right) \alpha_{\mathbf{n}, 1}(x)} d \sigma(x)=\frac{\alpha_{\mathbf{n}, 2}\left(x_{\mathbf{n}, j}\right)}{\alpha_{\mathbf{n}, 1}\left(x_{\mathbf{n}, j}\right)} S_{\mathbf{n}, N-1}\left(x_{\mathbf{n}, j}\right) \lambda_{\mathbf{n}, 1, j} .
\end{gathered}
$$

From (2.9), (3.1) and (3.5) we have that

$$
\left|\lambda_{\mathbf{n}, 2, j}\right| \leq \frac{1}{\sqrt{C_{1}}}\left|S_{\mathbf{n}, N-1}\left(x_{\mathbf{n}, j}\right)\right|\left(\frac{\lambda_{\mathbf{n}, 1, j}}{\alpha_{\mathbf{n}, 1}\left(x_{\mathbf{n}, j}\right)}\right)^{1 / 2} \lambda_{\mathbf{n}, 1, j}^{1 / 2}
$$

Summing term by term both sides of (3.6) and applying the interpolation condition of the quadrature formula and the Cauchy-Schwartz inequality we obtain that

$$
\begin{gathered}
\sum_{j=1}^{N}\left|\lambda_{\mathbf{n}, 2, j}\right| \leq \frac{1}{\sqrt{C_{1}}}\left(\sum_{j=1}^{N} \frac{S_{\mathbf{n}, N-1}^{2}\left(x_{\mathbf{n}, j}\right) \lambda_{\mathbf{n}, 1, j}}{\alpha_{\mathbf{n}, 1}\left(x_{\mathbf{n}, j}\right)}\right)^{1 / 2} M_{1} \\
=\frac{1}{\sqrt{C_{1}}}\left(\int_{a}^{b} \frac{S_{\mathbf{n}, N-1}^{2}(x)}{\alpha_{\mathbf{n}, 1}(x)} w_{1}(x) d \sigma(x)\right)^{1 / 2} M_{1} \\
\leq \frac{1}{\sqrt{C_{1}}}\left(\int_{a}^{b} \frac{\alpha_{\mathbf{n}, 1}(x) w_{2}^{2}(x)}{\alpha_{\mathbf{n}, 2}^{2}(x) w_{1}(x)} d \sigma(x)\right)^{1 / 2} M_{1} \leq \sqrt{\frac{C_{2} C_{0}}{C_{1}}} M_{1},
\end{gathered}
$$

where $M_{1}=\left(\sum_{j=1}^{N} \lambda_{\mathbf{n}, 1, j}\right)^{1 / 2}$ 口 
4. Quadratures formulas for Nikishin systems. A sequence of simultaneous quadrature formulas of rational type must be constructed by using multi-indexes $\mathbf{n}=\left(n_{1}, \ldots, n_{m}\right)$ which are strongly normal with respect to the given weights. The following theorem establishes a method for finding this multi-indexes and weights.

THEOREM 4.1. Let $S=\left\{w_{1}, \ldots, w_{m}\right\}$ be an AT-system for $\mathbf{n}=\left(n_{1}, \ldots n_{m}\right)$ on the interval $I=[a, b]$. Then $\mathbf{n}$ is strongly normal for $S$

Theorem 4.1 is a consequence of the theory of T-systems. For the proof we refer the reader to [19].

A well known example of $A T$-system is $S=\left\{\exp \left(\beta_{1} x\right), \ldots, \exp \left(\beta_{m} x\right)\right\}$, where $\beta_{1}<\cdots<\beta_{m}$. Other examples are given in the form of the so-called Nikishin systems (cf. [19]). Let $\sigma_{1}$ and $\sigma_{2}$ be two finite measures of constant sign on the intervals $I_{1}$ and $I_{2}$, respectively. If $I_{1} \cap I_{2}=\emptyset$ then we may define a non-commutative product

$$
\left\langle\sigma_{1}, \sigma_{2}\right\rangle(x)=\int_{I_{1}} \frac{d \sigma_{2}(t)}{x-t} d \sigma_{1}(x)=\widehat{\sigma}_{2}(x) d \sigma_{1}(x),
$$

which defines a new measure of constant sign on $I_{1}$. Let $\left\{\sigma_{1}, \ldots, \sigma_{m}\right\}$ be a system of measures on the intervals $I_{k}, k=1, \ldots, m$, respectively, and such that $I_{i} \cap I_{i-1}=\emptyset$, $i=1, \ldots, m-1$. By induction we define

$$
s_{1}=\sigma_{1}, s_{k}=\left\langle\sigma_{1},\left\langle\sigma_{2}, \ldots, \sigma_{k}\right\rangle\right\rangle .
$$

Thus, $\left(s_{i}\right)_{i=1, m}$ is a new system of measures all of them on the same interval $I_{1}$, usually called Nikishin system. The notation is $\left(s_{1}, \ldots, s_{m}\right)=\mathcal{N}\left(\sigma_{1}, \ldots, \sigma_{m}\right)$. A result of [19] is that all multi-indices of the form $(s, \ldots, s, s+1, \ldots, s+1)$ are strongly normal for $\mathcal{N}\left(\sigma_{1}, \ldots, \sigma_{m}\right)$. More generally, if $i<j \leq m$ implies $n_{j} \leq n_{i}+1$, the corresponding index $n$ is strongly normal for $\mathcal{N}\left(\sigma_{1}, \ldots, \sigma_{m}\right)$ (cf. [5]). Other examples of strongly normal multi-indices for a Nikishin system is given by the class $\mathbb{Z}_{+}^{m}(*)$ formed by those $\mathbf{n}=\left(n_{1}, \ldots, n_{m}\right)$ for which there are not $i, j, k$, with $1 \leq i<j<k \leq m$, such that $n_{i}<n_{j}<n_{k}$ (cf. [2]).

In this section we denote by $\alpha_{\mathbf{n}}$ a polynomial with real coefficients and

$$
\operatorname{deg} \alpha_{\mathbf{n}}<|\mathbf{n}|+\min \left\{n_{1}, \ldots, n_{m}\right\}-1,
$$

and such that $\left\{z \in \mathbb{C} ; \alpha_{\mathbf{n}}(z)=0\right\} \subset F \subset \overline{\mathbb{C}} \backslash I_{1}$, where $F$ is a compact set.

A rational modification of a Nikishin system can be easily obtained as follows.

Given the standard statement

$$
\left(\sigma_{1}, w_{2} \sigma_{1}, \ldots, w_{m} \sigma_{1}\right)=\mathcal{N}\left(\sigma_{1}, \sigma_{2}, \ldots, \sigma_{m}\right)
$$

then we only have to modified the first measure $\sigma_{1}$ to obtain

$$
\left(\frac{\sigma_{1}}{\alpha_{\mathbf{n}}}, \frac{w_{2} \sigma_{1}}{\alpha_{\mathbf{n}}}, \ldots, \frac{w_{m} \sigma_{1}}{\alpha_{\mathbf{n}}}\right)=\mathcal{N}\left(\frac{\sigma_{1}}{\alpha_{\mathbf{n}}}, \sigma_{2}, \ldots, \sigma_{m}\right),
$$

where the weights $w_{k}, k=2, \ldots, m$, are formed by nested Cauchy transforms according to equation (4.1) and (4.2).

THEOREM 4.2. Let $\Lambda \subset \mathbb{Z}_{+}^{m}$ be a sequence of strongly normal multi-indices for $\left(S,\left(\alpha_{\mathbf{n}}\right)_{\mathbf{n} \in \Lambda}\right)$ and that $\sup _{\mathbf{n} \in \Lambda}|\mathbf{n}|=\infty$. Let $S=\left(s_{1}, \ldots, s_{m}\right)=\mathcal{N}\left(\sigma_{1}, \ldots, \sigma_{m}\right)$ be a Nikishin system and let $\mathbf{n}=\left(n_{1}, \ldots, n_{m}\right) \in \Lambda$ be fixed. Given $M=\max \left\{n_{1}-1, n_{2}-\right.$ $\left.2, \ldots, n_{m}-2\right\}$ we select $k=k(\mathbf{n}) \in\{1, \ldots, m\}$ according to the following criterion: 
$k=1$ if $n_{1}-1=M$, or $k \in\{2, \ldots, m\}$ corresponds to the first component of $\mathbf{n}$ such that $n_{k}-2=M$. There exists a monic polynomial $W_{\mathbf{n}, k}$ with degree $|\mathbf{n}|-n_{k}$ and simple zeros in the interior of $I_{2}$ such that

$$
\begin{gathered}
\int_{I_{k}} x^{\nu} Q_{\mathbf{n}}(x) \frac{d s_{k}(x)}{\alpha_{\mathbf{n}}(x) W_{\mathbf{n}, k}(x)}=0, \nu=0,1, \ldots,|\mathbf{n}|-1 \\
\int_{I_{k}} \frac{p(x)}{\left(\alpha_{\mathbf{n}} W_{\mathbf{n}, k}\right)(x)} d s_{k}(x)=\sum_{j=1}^{|\mathbf{n}|} \lambda_{\mathbf{n}, k, j} \frac{p\left(x_{\mathbf{n}, j}\right)}{\left(\alpha_{\mathbf{n}} W_{\mathbf{n}, k}\right)\left(x_{\mathbf{n}, j}\right)}, p \in \Pi_{2|\mathbf{n}|-1} .
\end{gathered}
$$

For each $j=1, \ldots,|\mathbf{n}|$, the following equation takes place

$$
\lambda_{\mathbf{n}, k, j}=\left(\alpha_{\mathbf{n}} W_{\mathbf{n}, k}\right)\left(x_{\mathbf{n}, j}\right) \int_{I_{k}}\left(\frac{Q_{\mathbf{n}}(x)}{Q_{\mathbf{n}}^{\prime}\left(x_{\mathbf{n}, j}\right)\left(x-x_{\mathbf{n}, j}\right)}\right)^{2} \frac{d s_{k}(x)}{\left(\alpha_{\mathbf{n}} W_{\mathbf{n}, k}\right)(x)} .
$$

Proof. We apply ([8], Theorem 3) to the new Nikishin system given by (4.4).

Corollary 4.3. Let $S=\left(s_{1}, \ldots, s_{m}\right)=\mathcal{N}\left(\sigma_{1}, \ldots, \sigma_{m}\right)$ be a Nikishin system and let $k \in\{1, \ldots, m\}$ be fixed. Let $\Lambda \subset \mathbb{Z}_{+}^{m}(*)$ be an infinite sequence of distinct multiindexes $\mathbf{n}=\left(n_{1}, \ldots, n_{m}\right)$ such that for all $\mathbf{n} \in \Lambda$ the $k$-th component $n_{k}$ satisfies the hypothesis of Theorem 4.2. Let $\mathbf{T}=\left\{z_{j} ; j \in \mathbb{N}\right\}$ be a table of real numbers such that $\left|z_{j}\right|>1, j \in \mathbb{N}$. If $\mathbf{T}$ has the density property with respect to $I_{1}$ and $\left\{\alpha_{\mathbf{n}} ; \mathbf{n} \in \Lambda\right\}$ is the associated sequence of polynomials whose degree fulfills (4.3), then the $k$-th rational quadrature formula of the corresponding simultaneous system converges for all bounded Riemann integrable functions on $I_{1}$.

Proof. From Theorem 4.2 we have that coefficients $\lambda_{\mathbf{n}, k, j}, \mathbf{n} \in \Lambda$, fulfills conditions A) and B) (Section 2). More precisely, all the weights $\lambda_{\mathbf{n}, k, j}$ have the same sign as the measure $s_{k}$ and

$$
\sum_{j=1}^{|\mathbf{n}|}\left|\lambda_{\mathbf{n}, k, j}\right|=\left|s_{k}\right|
$$

The corollary follows from Proposition 2.8.

REMARKS 4.

1. The sequence $\Lambda \subset \mathbb{Z}_{+}^{m}(*)$ defined by $n_{j}=N, j=1, \ldots, k-1$, and $n_{j}=N+1$, $j=k, \ldots, m, N \in \mathbb{N}$ satisfies the hypothesis of Theorem 4.2 in the first and the $k$-th component.

2. The criterion which we have followed to choose a suitable $k$ in Theorem 4.2 does not admit more than two values for $k$. For the moment we can only prove for every rule of the simultaneous system, that the majority of the NikishinChristoffel coefficients have the same sign as the corresponding measure.

Definition 4.4. Let $\mathbf{n}=\left(n_{1}, \ldots, n_{m}\right) \in \mathbb{Z}_{+}^{m}$. For every $i=1, \ldots, m$, we construct an associated multi-index $\mathbf{n}^{i}=\left(n_{1}^{i}, \ldots, n_{m}^{i}\right) \in \mathbb{Z}_{+}^{m-1}$, in the following form (cf. [8])

$$
n_{j}^{i}= \begin{cases}\min \left\{n_{1}, \ldots, n_{j-1}, n_{i}-1\right\} & j=2, \ldots, i \\ \min \left\{n_{i}, \ldots, n_{j}\right\} & j=i+1, \ldots, m .\end{cases}
$$


THEOREM 4.5. Let $S=\left(s_{1}, \ldots, s_{m}\right)=\mathcal{N}\left(\sigma_{1}, \ldots, \sigma_{m}\right)$ be a Nikishin system. For $\mathbf{n} \in \mathbb{Z}_{+}^{m}$ let $\mathbf{n}^{i}$ be given as that in Definition 4.4. Then, for every $i \in\{1, \ldots, m\}$ there exists a monic polynomial $W_{\mathbf{n}, i}$ of degree $\left|\mathbf{n}^{i}\right|$ whose zeros are simple and lie in the interior of $I_{2}$, such that

$$
\int_{I_{i}} \frac{p(x)}{\left(\alpha_{\mathbf{n}} W_{\mathbf{n}, i}\right)(x)} d s_{i}(x)=\sum_{j=1}^{|\mathbf{n}|} \lambda_{\mathbf{n}, i, j} \frac{p\left(x_{\mathbf{n}, j}\right)}{\left(\alpha_{\mathbf{n}} W_{\mathbf{n}, i}\right)\left(x_{\mathbf{n}, j}\right)},
$$

$p \in \Pi_{|\mathbf{n}|+\left|\mathbf{n}^{i}\right|+n_{i}-1}$.

Moreover, at least $\left(|\mathbf{n}|+\left|\mathbf{n}^{i}\right|+n_{i}\right) / 2$ coefficients $\lambda_{\mathbf{n}, i, j}$ have the same sign as the measure $s_{i}$.

Proof. The existence of the polynomial $W_{\mathbf{n}, i}$ follows from ([7], Theorem 2 and 3 ). Moreover, $W_{\mathbf{n}, i}$ satisfies the following orthogonality relations.

$$
0=\int x^{\nu} Q_{\mathbf{n}}(x) \frac{d s_{i}(x)}{\left(\alpha_{\mathbf{n}} W_{\mathbf{n}, i}\right)(x)}, \nu=0, \ldots,\left|\mathbf{n}^{i}\right|+n_{i}-1,
$$

which proves (4.10).

Let $\tau_{\mathbf{n}}$ be the number of indices $j$ such that $\operatorname{sign}\left(\lambda_{\mathbf{n}, i, j}\right)=\operatorname{sign}\left(s_{i}\right)$. Select $p_{1}(x)=$ $\Pi^{\prime}\left(x-x_{\mathbf{n}, j}\right)^{2}$, where $\prod^{\prime}$ denotes the product over all the indices $j$ such that $\operatorname{sign}\left(\lambda_{\mathbf{n}, i, j}\right)=$ $\operatorname{sign}\left(s_{i}\right)$. Let us assume that $\operatorname{deg} p=2 \tau_{\mathbf{n}} \leq|\mathbf{n}|+\left|\mathbf{n}^{i}\right|+n_{i}-1$, then (4.10) yields

$$
\operatorname{sign}\left(\int_{I_{i}} \frac{p_{1}(x)}{\left(\alpha_{\mathbf{n}} W_{\mathbf{n}, i}\right)(x)} d s_{i}(x)\right) \neq \operatorname{sign}\left(\sum_{j=1}^{|\mathbf{n}|} \lambda_{\mathbf{n}, i, j} \frac{p_{1}\left(x_{\mathbf{n}, j}\right)}{\left(\alpha_{\mathbf{n}} W_{\mathbf{n}, i}\right)\left(x_{\mathbf{n}, j}\right)}\right),
$$

which is a contradiction. Theorem (4.5) is proved.

REMARKS 5. A convergence result analogous to Corollary 4.3 can be obtained for sequences of multi-indexes of the form $n=(N, \ldots, N, N+1, \ldots, N+1)$, where $n_{k^{\prime}-1}=N$ and $n_{k^{\prime}}=N+1$, and $k^{\prime}$, the component where the jump occurs, is fixed. In this case, for $k=1, k^{\prime}, k^{\prime}+1$, the $k$-th rational simultaneous quadrature formula converges for all bounded and Riemann integrable function on $I_{1}$. The polynomial version of Corollary 4.3 is given in ([8], Corollary 3).

5. The numerical method. In what follows we adopt a notation for matrices inspired in Matlab language. We only consider the case $m=3$ and the sequence of multi-indices

$$
n(r)=\left\{\begin{array}{ll}
(s, s, s) & r=3 s \\
(s, s, s+1) & r=3 s+1 \\
(s, s+1, s+1) & r=3 s+2
\end{array} \quad s=0,1,2, \ldots\right.
$$

Notice that $n(r) \in \mathbb{Z}_{+}^{3}(*), r=0,1,2, \ldots$

Let $w_{k}(x), k=1,2,3$, be three weight functions on the interval $[a, b]$ which forms an $A T$-system. Let $\mu$ be a measure with constant sign on $[a, b]$. Given $n(r)$ let $Q_{r}$ be the $r$-th multi-orthogonal polynomial associated to $(S, \alpha)$, where $S=\left\{w_{1}, w_{2}, w_{3}\right\}$ and $\alpha$ is a suitable polynomial.

It is well known that $\left\{Q_{r}\right\}$ satisfies a recurrence formula of $m+2=5$ terms of the following type.

$$
Q_{r}=\left(x-a_{r}\right) Q_{r-1}-b_{r} Q_{r-2}-c_{r} Q_{r-3}-d_{r} Q_{r-4}, \quad r=1,2, \ldots
$$


Let $z(j) \in \mathbb{R} \backslash[a, b], j=1, \ldots, K$, and $\zeta(j) \in \mathbb{R}, j=1, \ldots, s$, be the zeros of the polynomials $\alpha$ and $P_{s}$, respectively, that is

$$
\alpha(x)=\frac{x-z(1)}{h_{\alpha}-z(1)} \cdots \frac{x-z(K)}{h_{\alpha}-z(K)}, \quad P_{s}(x)=\frac{x-\zeta(1)}{h_{P}-\zeta(1)} \cdots \frac{x-\zeta(s)}{h_{P}-\zeta(s)},
$$

where the array $z(\cdot)$ simulates the most difficult poles of a given integrand and the parameters $h_{\alpha}, h_{P}$ are conveniently chosen.

Let $\left\{q_{k}(s, r)\right\}$ be a table defined as

$$
q_{k}(s, r)=\int_{a}^{b} Q_{r}(x) P_{s}(x) \frac{w_{k}(x)}{\alpha(x)} d \mu(x), r, s=0,1,2, \ldots, k=1,2,3
$$

Let $E_{k}\left(s, r, a_{r}, b_{r}, c_{r}, d_{r}\right)$ be the following expression

$$
\begin{gathered}
E_{k}\left(s, r, a_{r}, b_{r}, c_{r}, d_{r}\right)=\left(h_{P}-\zeta(s)\right) q_{k}(s+1, r)+\zeta(s) q_{k}(s, r)-a_{r} q_{k}(s, r)- \\
b_{r} q_{k}(s, r-1)-c_{r} q_{k}(s, r-2)-d_{r} q_{k}(s, r-3), \quad k=1,2,3 .
\end{gathered}
$$

From (5.2) we obtain the following relation

$$
E_{k}\left(s, r, a_{r}, b_{r}, c_{r}, d_{r}\right)=q_{k}(s, r+1)
$$

Let $\mathcal{X}_{r}=\left[a_{r}, b_{r}, c_{r}, d_{r}\right]^{T}$. In what follows we will write $E_{k}\left(s, r, \mathcal{X}_{r}\right)$ or simply $E_{k}(s, r)$ when explicit mention of $\mathcal{X}_{r}$ is not relevant.

The vector of coefficients $\mathcal{X}_{r}$ is the solution of a basic linear system of equations $\mathcal{A}_{r} \mathcal{X}_{r}=\mathcal{B}_{r}$ whose construction depends on the value of $r$. If $r=3 s$ the system is formed by equations $E_{k}(s-1, r-1)=0, k=1,2,3$, and $E_{1}(s-2, r-1)=0$. If $r=3 s+1$ then the equations are $E_{3}(s, r-1)=0$ and $E_{k}(s-1, r-1)=0, k=1,2,3$. Finally, for $r=3 s+2$ we get $E_{k}(s, r-1)=0, k=2,3$, and $E_{k}(s-1, r-1)=0$, $k=1,2$.

Our computational strategy is based on two principles: I) extensive use of data, and II) a well balanced participation of the three integration weights in calculations. Mathematically speaking, for every $r$ we only need the four equations mentioned above to determine the solution $\mathcal{X}_{r}$. However, errors occur due to the arithmetic of finite precision what could be made worse because of the complexity of some weights functions. We claim that accuracy may be improved by adding more equations from above and/or below the basic systems. For example, for $r=3 s$ the first equation from above is $E_{3}(s, 3 s-1)=q_{3}(s, 3 s)$. In general, all equations from above the basic systems have the form (5.5) because the term $q_{k}(s, r+1)$ is different from zero, thus one needs a prediction $q_{k}^{(0)}(s, r+1)$.

On the other hand, all equations from below the basic systems have zero coefficients. We denote by $\mathcal{A}_{r}^{\prime} \mathcal{X}_{r}=\mathcal{B}_{r}^{\prime}$ any system formed by equations from above any basic system of equations $\mathcal{A}_{r} \mathcal{X}_{r}=\mathcal{B}_{r}$.

Once we have found the vector $\mathcal{X}_{r}$ we can construct matrices $M_{j}, j \geq r$ up to the $r$-th row. The eigenvalues of $M_{r}$, whose structure is determined by equation (5.2), 
are the zeros of $Q_{r}$. Below we outline the form of $M_{r}$.

$$
M_{r}=\left(\begin{array}{cccccccccccccc}
a_{1} & 1 & 0 & 0 & 0 & 0 & 0 & 0 & \cdots & 0 & 0 & 0 & 0 & 0 \\
b_{2} & a_{2} & 1 & 0 & 0 & 0 & 0 & 0 & \cdots & 0 & 0 & 0 & 0 & 0 \\
c_{3} & b_{3} & a_{3} & 1 & 0 & 0 & 0 & 0 & \cdots & 0 & 0 & 0 & 0 & 0 \\
d_{4} & c_{4} & b_{4} & a_{4} & 1 & 0 & 0 & 0 & \cdots & 0 & 0 & 0 & 0 & 0 \\
0 & d_{5} & c_{5} & b_{5} & a_{5} & 1 & 0 & 0 & \cdots & 0 & 0 & 0 & 0 & 0 \\
\vdots & & & & & & & & \ddots & & & & & \vdots \\
0 & 0 & 0 & 0 & 0 & 0 & 0 & 0 & \cdots & 0 & d_{r} & c_{r} & b_{r} & a_{r}
\end{array}\right)
$$

According to the so-called bootstrapping method, the next step consists in calculating $\mathcal{A}_{r+1}$ and $\mathcal{B}_{r+1}$ to obtain $Q_{r+1}$ and its zeros as the eigenvalues of $M_{r+1}$. Indeed, the explicit expression of polynomials $Q_{r}$ is not needed.

A lot of computational effort must be carried out to avoid loss of accuracy when the zeros of $\alpha(x)$ are too close to the integration interval $[a, b]$. Special techniques to treat these difficult poles have been developed by Gautschi [9, 10] (see also [17]). Nevertheless, our method is not based on Gautschi's but on the use of some transformations of the integration interval. The latter approach is also known as the "change of variable method" because it is exactly what we do before applying the integration rule. If $\varphi$ transforms $[a, b]$ onto itself and $\varphi^{\prime}>0$, then

$$
\int_{a}^{b} f(x) d x=\int_{a}^{b} f(\varphi(x)) \varphi^{\prime}(x) d x .
$$

Given any integration rule with nodes $x_{k} \in[a, b]$, and weights $\lambda_{k}, k=1, \ldots, r$, it can be applied to the right integral in (5.6) instead of the left one. This process yields a new rule with nodes $y_{k}=\varphi\left(x_{k}\right)$, and weights $\Lambda_{k}=\lambda_{k} \varphi^{\prime}\left(x_{k}\right)$ to evaluate the left integral in (5.6). Our choice is $(a<b)$

$$
\varphi_{p, q, a, b}(x)=\frac{(b-a)(x-a)^{p}}{(x-a)^{p}+(b-x)^{q}}+a ; p, q \in \mathbb{N} q, p \geq 1, p+q>2,
$$

which transforms $[a, b]$ onto $[a, b]$.

Transformations (5.7) have played a major role in solving certain kind of singular integral equations. The non-symmetric case in (5.7), which occurs when $p \neq q$, was promoted by Monegato \& Scuderi when the integrand has only one end-point singularity (see [18] and the bibliography therein).

From both numerical and theoretical points of view, interpolatory rational quadratures formulas are nothing else than product integration rules. Possibly their main disadvantage is that every collection of difficult poles requires a specific set of nodes and weights. Gautschi $[9,10]$ suggested that the zeros of $\alpha$ only have to approximate poles but he did not establish how much. Thus, one of the most important tasks in this context is the design of low cost algorithms to calculate these formulas according to which poles must be simulated.

The main stages of the procedure are summarized in the following items.

1. Calculation of the modified moments $q_{k}(s, 0), s=0, \ldots, s_{0}$, where the size of $s_{0}$ depends on what is the maximum quadrature order $r$ to be reached, v.g. $24 \leq s_{0} \leq 30$ for $r \leq 12$.

2. Calculate $\mathcal{X}_{1}=a_{1}=\mathcal{A}_{1} / \mathcal{B}_{1}$ and $q_{k}(s, 1), s=0, . ., s_{0}-1$, where

$$
\mathcal{A}_{1}=q_{3}(1,0), \quad \mathcal{B}_{1}=\left(h_{P}-\zeta(1)\right) * q 3(1,0)+\zeta(1) * q 3(0,0) .
$$


3. Calculate $\mathcal{X}_{2}=\left[a_{2}, b_{2}\right]^{T}=\mathcal{A}_{2} \backslash \mathcal{B}_{2}$ and $q_{k}(s, 2), s=0, . ., s_{0}-2$, where $\mathcal{A}_{2}(k,:)=\left[q_{k}(0,1), q_{k}(0,0)\right], \quad \mathcal{B}_{2}(k, 1)=\left(h_{P}-\zeta(1)\right) * q_{k}(1,1)+\zeta(1) * q_{k}(0,1)$, $k=1,2$.

4. Calculate $\mathcal{X}_{3}=\left[a_{3}, b_{3}, c_{3}\right]^{T}=\mathcal{A}_{3} \backslash \mathcal{B}_{3}$ and $q_{k}(s, 2), s=0, . ., s_{0}-3$, where

$$
\begin{gathered}
\mathcal{A}_{3}(k,:)=\left[q_{k}(0,2), q_{k}(0,1), q_{k}(0,0)\right], \\
\mathcal{B}_{3}(k, 1)=\left(h_{P}-\zeta(1)\right) * q_{k}(1,2)+\zeta(1) * q_{k}(0,2), k=1,2,3
\end{gathered}
$$

5. Given the vector $\mathcal{X}_{r}=\left[a_{r}, b_{r}, c_{r}, d_{r}\right]^{T}, r \geq 4$, and $q_{k}\left(0: s_{0}-r+1,0: r\right)$, $k=1,2,3$, calculate the vector $\mathcal{X}_{r+1}=\left[a_{r+1}, b_{r+1}, c_{r+1}, d_{r+1}\right]^{T}$ from the system $\mathcal{A}_{r+1} \mathcal{X}_{r+1}=\mathcal{B}_{r+1}$, and $q_{k}\left(0: s_{0}-r, r+1\right), k=1,2,3$ from (5.4).

The quadrature nodes $\left(x_{\mathbf{n}, j}\right)_{j=1}^{r}$ are computed in the $r$-th step as the eigenvalues of the matrix $M_{r}$.

The vector of coefficients $\Lambda_{k, r}=\left[\lambda_{\mathbf{n}, k, 1}, \ldots, \lambda_{\mathbf{n}, k, r}\right]^{T}$ fulfills

$$
\Lambda_{k, r}=\operatorname{dot}\left(\eta_{k, r},\left[w_{k}\left(x_{\mathbf{n}, 1}\right), \ldots, w_{k}\left(x_{\mathbf{n}, r}\right)\right]\right),
$$

where $\eta_{k, r}$ is the solution of the over-determined system $V_{r} \eta_{k, r}=D_{k, r}$, with

$$
V_{r}=\left[v\left(0: r+n_{k}-1,1: r\right)\right]
$$

$v(i, j)=P_{i}\left(x_{\mathbf{n}, j}\right)$, and $D_{k, r}=\left[q_{k}\left(0: r+n_{k}-1,0\right)\right]$.

Rounding errors, which occur in evaluating $q_{k}(s, 0)$, cause an increasingly large perturbation in $q_{k}(s, r)$ as $r$ increases. Other important source of error is that produced by the presence of poles in the integrand. Those poles considered as difficult ones yield slow convergence and loss of accuracy. We overcome this drawback by using transformation (5.7) and Gauss-Legendre quadrature rule.

The next subsections deal with two different AT-systems, the second one arises from Nikishin's method. For the purposes of comparison we have selected two examples from [9] for which the first integrating measure is $w_{1}(x) d \mu(x)=d x$.

5.1. Test 1. The integrals to be evaluated are

$$
I_{k}=\int_{-1}^{1} \frac{\pi x}{\omega \sin (\pi x / \omega)} w_{k}(x) d \mu(x), \omega>0, k=1,2,3
$$

where $d \mu(x)=d x, w_{1}(x)=1, w_{2}(x)=\exp (x)$ and $w_{3}(x)=\exp (x \sqrt{3})$. It is well known that this system of weight functions forms an AT-system (cf. [19]).

The integrand in (5.8) is analytic in a neighborhood of the interval $[-1,1]$, and has real poles in $N \omega, N \in \mathbb{Z}, N \neq 0$. When $\omega$ is small the most difficult poles must be considered by pairs $\xi_{N}= \pm N \omega, N=1, \ldots, d$, where $d$ is selected according to the size of $\omega$. In order to simulate the $K$ poles closest to $[-1,1]$ we will assume that polynomials $\alpha(x)$ have zeros at: $\pm j \omega, j=1, \ldots d$, hence $K=2 d$. The zero $\omega(d+1)$ is included in case of $K=2 d+1$. We conclude that $\alpha$ has one of the two forms given below.

$$
\alpha_{2 d}(x)=\prod_{k=1}^{d}\left(\frac{x^{2}-1}{1-(k \omega)^{2}}+1\right), \quad \alpha_{2 d-1}(x)=\left(\frac{x-1}{1-d \omega}+1\right) \alpha_{2 d-2}(x) .
$$


TABLE 5.1

Relative errors obtained in Test 1 when the integrals $I_{i}(1.001), i=1,2,3$, are evaluated by a simultaneous rational rule $\left(S R Q F^{(1)}\right)$

\begin{tabular}{c|ccc|ccc}
$w_{k}$ & $r$ & $\operatorname{deg} \alpha$ & SRQF $^{(1)}$ & $r$ & $\operatorname{deg} \alpha$ & SRQF $^{(1)}$ \\
\hline 1 & 1 & 2 & $8.3 \mathrm{e}-02$ & 7 & 2 & $1.6 \mathrm{e}-07$ \\
2 & & & $1.1 \mathrm{e}-01$ & & & $3.9 \mathrm{e}-08$ \\
3 & & & $1.3 \mathrm{e}-01$ & & & $6.1 \mathrm{e}-08$ \\
\hline 1 & 2 & 2 & $8.5 \mathrm{e}-02$ & 8 & 2 & $1.2 \mathrm{e}-08$ \\
2 & & & $1.8 \mathrm{e}-02$ & & & $2.9 \mathrm{e}-09$ \\
3 & & & $1.6 \mathrm{e}-02$ & & & $4.6 \mathrm{e}-09$ \\
\hline 1 & 3 & 2 & $3.7 \mathrm{e}-03$ & 9 & 2 & $7.3 \mathrm{e}-10$ \\
2 & & & $1.1 \mathrm{e}-03$ & & & $2.2 \mathrm{e}-10$ \\
3 & & & $2.1 \mathrm{e}-03$ & & & $3.9 \mathrm{e}-10$ \\
\hline 1 & 4 & 2 & $4.0 \mathrm{e}-04$ & 10 & 2 & $6.1 \mathrm{e}-11$ \\
2 & & & $8.9 \mathrm{e}-05$ & & & $1.5 \mathrm{e}-11$ \\
3 & & & $1.3 \mathrm{e}-04$ & & & $2.5 \mathrm{e}-11$ \\
\hline 1 & 5 & 2 & $3.3 \mathrm{e}-05$ & 11 & 2 & $6.9 \mathrm{e}-12$ \\
2 & & & $7.2 \mathrm{e}-06$ & & & $7.9 \mathrm{e}-13$ \\
3 & & & $1.0 \mathrm{e}-05$ & & & $4.2 \mathrm{e}-13$ \\
\hline 1 & 6 & 2 & $1.8 \mathrm{e}-06$ & 12 & 2 & $1.2 \mathrm{e}-12$ \\
2 & & & $5.7 \mathrm{e}-07$ & & & $1.5 \mathrm{e}-13$ \\
3 & & & $1.0 \mathrm{e}-06$ & & & $6.5 \mathrm{e}-13$ \\
\hline
\end{tabular}

Some results are shown in Table 5.1, 5.2 and 5.3 when $\omega=1.001$.

Fast convergence of our procedure is appraised in Table 5.1, where the relative errors for the first order values $r=1, \ldots, 12$ are listed, and $\operatorname{deg} \alpha=2$.

The values of $q_{k}(s, 0), s=0, \ldots, 34$ are computed with a relative error about $1.0 e-$ 15 by using composite Gauss-Legendre formula and the smoothing transformations (5.7) with $p=q=4$.

Relative errors when we applied simultaneous quadrature formulas of polynomial $(\mathrm{SPQF}, K=0)$ and rational type $\left(\mathrm{SRQF}^{(1)}\right)$ are compared in Table 5.2.

A comparison with the numerical results in [9] is shown in Table 5.3 where selected output is organized. Here the errors are produced by a Gauss rational quadrature formula (RGQF) and a simultaneous rational quadrature formula $\left(\mathrm{SRQF}^{(1)}\right)$ when the measure is $w_{1}(x) d \mu(x)=d x$. If $\operatorname{deg} \alpha=K$ then $\max \{r-K, 0\}$ indicates polynomial participation in a mixed procedure. Numerical results in column RGQF were extracted from [9].

5.2. Test 2. Let $\left(d s_{1}, d s_{2}, d s_{3}\right)=\mathcal{N}\left(d \sigma_{1}, d \sigma_{2}, d \sigma_{3}\right)$, where $d \sigma_{1}(x)=d x / \sqrt{x}$, $x \in[0,1], d \sigma_{2}(x)=x^{2} d x, x \in[2,3]$, and $d \sigma_{3}(x)=d x, x \in[4,5]$. Then $d s_{1}=d \sigma_{1}$,

$d s_{2}(x)=\left(-2.5-x-x^{2} \log \left(\frac{3-x}{2-x}\right)\right) \frac{d x}{\sqrt{x}}, \quad d s_{3}(x)=\left(\int_{2}^{3} \log \left(\frac{4-s}{5-s}\right) \frac{s^{2} d s}{x-s}\right) \frac{d x}{\sqrt{x}}$.

Next we describe the experiment of calculating simultaneously the following integrals.

$$
J_{i}(\omega)=\int_{0}^{1} \frac{\Gamma(1+x)}{x+\omega} d s_{i}(x), \quad i=1,2,3 .
$$

The integral $J_{1}(\omega)$ is evaluated in Gautschi [9] (example 4.2) using a RGQF, and a discretization procedure for the modified inner product based on the recursion coefficients of the Jacobi polynomials with parameters $\alpha=0, \beta=-1 / 2$.

The common integrand in (5.9) has poles at $\omega$ and at $x=-1-j, j \in \mathbb{N}$. The non polar singularity at $x=0$ produced by $\sqrt{x}$ in the denominator is here considered as 
TABLE 5.2

Relative errors obtained in Test 1 when the integrals $I_{i}(1.001), i=1,2,3$, are evaluated by simultaneous integration rules of rational and polynomial type

\begin{tabular}{|c|c|c|c|c|c|}
\hline & \multicolumn{3}{|r|}{ Rational } & \multicolumn{2}{|c|}{ Polynomial } \\
\hline$w_{k}$ & $r$ & $\operatorname{deg} \alpha$ & $\mathrm{SRQF}^{(1)}$ & $r$ & SPQF \\
\hline 1 & 2 & 4 & $6.8 \mathrm{e}-02$ & 2 & $7.2 \mathrm{e}-01$ \\
\hline 2 & & & $1.3 \mathrm{e}-02$ & & 7.1e-01 \\
\hline 3 & & & $1.1 \mathrm{e}-02$ & & $7.5 \mathrm{e}-01$ \\
\hline 1 & 4 & 4 & $2.6 \mathrm{e}-04$ & 4 & $5.3 \mathrm{e}-01$ \\
\hline 2 & & & $5.4 \mathrm{e}-05$ & & $5.4 \mathrm{e}-01$ \\
\hline 3 & & & $7.9 \mathrm{e}-05$ & & $5.8 \mathrm{e}-01$ \\
\hline 1 & 5 & 6 & $1.8 \mathrm{e}-05$ & 5 & $4.7 \mathrm{e}-01$ \\
\hline 2 & & & $3.7 \mathrm{e}-06$ & & $4.9 \mathrm{e}-01$ \\
\hline 3 & & & $5.5 \mathrm{e}-06$ & & $5.2 \mathrm{e}-01$ \\
\hline 1 & 6 & 6 & $9.6 \mathrm{e}-07$ & 6 & $4.2 \mathrm{e}-01$ \\
\hline 2 & & & $2.9 \mathrm{e}-07$ & & $4.4 \mathrm{e}-01$ \\
\hline 3 & & & $5.2 \mathrm{e}-07$ & & $4.7 \mathrm{e}-01$ \\
\hline 1 & 7 & 8 & $7.8 \mathrm{e}-08$ & 7 & $3.8 \mathrm{e}-01$ \\
\hline 2 & & & $1.8 \mathrm{e}-08$ & & $4.0 \mathrm{e}-01$ \\
\hline 3 & & & $2.9 \mathrm{e}-08$ & & $4.2 \mathrm{e}-01$ \\
\hline 1 & 8 & 8 & $5.8 \mathrm{e}-09$ & 8 & $3.5 \mathrm{e}-01$ \\
\hline 2 & & & $1.4 \mathrm{e}-09$ & & $3.6 \mathrm{e}-01$ \\
\hline 3 & & & $2.2 \mathrm{e}-09$ & & $3.8 \mathrm{e}-01$ \\
\hline 1 & 9 & 8 & $3.5 \mathrm{e}-10$ & 9 & $3.1 \mathrm{e}-01$ \\
\hline 2 & & & $1.0 \mathrm{e}-10$ & & $3.3 \mathrm{e}-01$ \\
\hline 3 & & & $1.9 \mathrm{e}-10$ & & $3.5 \mathrm{e}-01$ \\
\hline 1 & 10 & 8 & $3.0 \mathrm{e}-11$ & 10 & $2.9 \mathrm{e}-01$ \\
\hline 2 & & & $7.0 \mathrm{e}-12$ & & $3.0 \mathrm{e}-01$ \\
\hline 3 & & & $1.1 \mathrm{e}-11$ & & $3.2 \mathrm{e}-01$ \\
\hline 1 & 11 & 8 & $4.4 \mathrm{e}-12$ & 11 & $2.6 \mathrm{e}-01$ \\
\hline 2 & & & $1.4 \mathrm{e}-13$ & & $2.7 \mathrm{e}-01$ \\
\hline 3 & & & $6.2 \mathrm{e}-13$ & & $2.9 \mathrm{e}-01$ \\
\hline
\end{tabular}

TABLE 5.3

Relative errors obtained in Test 1 when $I_{1}(1.001)$ is evaluated by a simultaneous rational rule $\left(S R Q F^{(1)}\right)$, and by a rational Gaussian quadrature formula (RGQF)

\begin{tabular}{cccc|cccc}
\hline$r$ & $\operatorname{deg} \alpha$ & RGQF & SRQF $^{(1)}$ & $r$ & deg $\alpha$ & RGQF & SRQF $^{(1)}$ \\
\hline 3 & 2 & $4.1 \mathrm{e}-01$ & $3.7 \mathrm{e}-03$ & 5 & 4 & $2.7 \mathrm{e}-01$ & $2.1 \mathrm{e}-05$ \\
6 & 4 & $8.4 \mathrm{e}-03$ & $1.1 \mathrm{e}-06$ & 6 & 4 & $8.4 \mathrm{e}-03$ & $1.1 \mathrm{e}-06$ \\
9 & 6 & $1.1 \mathrm{e}-04$ & $3.7 \mathrm{e}-10$ & 7 & 4 & $3.4 \mathrm{e}-04$ & $9.8 \mathrm{e}-08$ \\
12 & 8 & $9.0 \mathrm{e}-06$ & $5.1 \mathrm{e}-13$ & 9 & 4 & $6.6 \mathrm{e}-06$ & $4.3 \mathrm{e}-10$ \\
& & & & 10 & 4 & $6.1 \mathrm{e}-06$ & $3.6 \mathrm{e}-11$ \\
2 & 1 & $4.1 \mathrm{e}-01$ & $3.8 \mathrm{e}-01$ & & & & \\
4 & 2 & $2.9 \mathrm{e}-02$ & $4.0 \mathrm{e}-04$ & 5 & 2 & $2.2 \mathrm{e}-03$ & $3.3 \mathrm{e}-05$ \\
6 & 3 & $2.1 \mathrm{e}-03$ & $8.1 \mathrm{e}-07$ & 6 & 2 & $1.8 \mathrm{e}-04$ & $1.8 \mathrm{e}-06$ \\
8 & 4 & $1.9 \mathrm{e}-05$ & $7.3 \mathrm{e}-09$ & 7 & 2 & $2.0 \mathrm{e}-05$ & $1.6 \mathrm{e}-07$ \\
10 & 5 & $7.3 \mathrm{e}-06$ & $2.3 \mathrm{e}-11$ & 8 & 2 & $9.3 \mathrm{e}-06$ & $1.2 \mathrm{e}-08$ \\
12 & 6 & $5.0 \mathrm{e}-06$ & $5.6 \mathrm{e}-13$ & 9 & 2 & $7.8 \mathrm{e}-06$ & $7.3 \mathrm{e}-10$ \\
\hline
\end{tabular}

part of the weight functions and, unlike ([9], example 4.2), it does not participate in the definition of the Gaussian quadrature formula to be applied in evaluating (5.3).

Once more we fit transformation (5.7) into the modified moments $q_{k}(s, 0)$-not into the target integrals (5.9). After that we apply Gauss-Legendre rule to evaluate them. Table 5.4 shows the relative error yielded by a $\mathrm{SQRF}^{(2)}$ when (5.7) is taken with $\omega=0.001, a=0, b=1, p=6, q=1$, and the integrating measures are now modified by $1 / \alpha(x)$, where $\alpha(x)=(x+\omega) \prod_{j=1}^{K}(x+j)$. 
TABLE 5.4

Relative errors obtained in Test 2 when the integrals $J_{i}(0.001), i=1,2,3$, with respect to the Nikishin AT-system $\mathcal{N}\left(d x / \sqrt{x}, x^{2} d x, d x\right)$, are evaluated by a simultaneous rational quadrature formula

\begin{tabular}{c|ccc|ccc}
\hline$w_{k}$ & $r$ & $\operatorname{deg} \alpha$ & SRQF $^{(2)}$ & $r$ & $\operatorname{deg} \alpha$ & SRQF $^{(2)}$ \\
\hline 1 & 2 & 1 & $1.666 \mathrm{e}-02$ & 6 & 3 & $2.153 \mathrm{e}-12$ \\
2 & & & $1.070 \mathrm{e}-02$ & & & $3.246 \mathrm{e}-12$ \\
3 & & & $1.084 \mathrm{e}-02$ & & & $3.388 \mathrm{e}-12$ \\
\hline 1 & 4 & 1 & $2.862 \mathrm{e}-05$ & 8 & 3 & $2.325 \mathrm{e}-14$ \\
2 & & & $1.730 \mathrm{e}-05$ & & & $5.974 \mathrm{e}-13$ \\
3 & & & $1.745 \mathrm{e}-05$ & & & $6.181 \mathrm{e}-13$ \\
\hline 1 & 6 & 1 & $4.093 \mathrm{e}-08$ & 4 & 4 & $2.428 \mathrm{e}-07$ \\
2 & & & $2.468 \mathrm{e}-08$ & & & $2.484 \mathrm{e}-07$ \\
3 & & & $2.490 \mathrm{e}-08$ & & & $2.710 \mathrm{e}-07$ \\
\hline 1 & 6 & 2 & $1.237 \mathrm{e}-10$ & 6 & 4 & $4.067 \mathrm{e}-12$ \\
2 & & & $6.058 \mathrm{e}-11$ & & & $2.588 \mathrm{e}-11$ \\
3 & & & $6.133 \mathrm{e}-11$ & & & $2.704 \mathrm{e}-11$ \\
\hline 1 & 8 & 2 & $1.326 \mathrm{e}-11$ & 8 & 4 & $2.991 \mathrm{e}-13$ \\
2 & & & $6.316 \mathrm{e}-12$ & & & $3.747 \mathrm{e}-13$ \\
3 & & & $6.390 \mathrm{e}-12$ & & & $3.887 \mathrm{e}-13$ \\
\hline 1 & 4 & 3 & $7.460 \mathrm{e}-08$ & 9 & 4 & $1.375 \mathrm{e}-15$ \\
2 & & & $1.843 \mathrm{e}-07$ & & & $1.179 \mathrm{e}-15$ \\
3 & & & $1.978 \mathrm{e}-07$ & & & $1.270 \mathrm{e}-15$ \\
\hline
\end{tabular}

The present AT-system requires routines strictly numeric to simulate the Cauchytype integrals which arise in the Nikishin's procedure. This restriction is due to the slow performance of symbolic tools. For that reason we have implemented several routines from which we found that those based on a quadrature formula seem to be the most stable. However, instability still shows up for $r \geq 7$, so the estimate of $q_{k}(s, r+1)$ obtained in step 5 should be improved. If $\mathcal{X}_{r}^{(0)}$ is the solution of $\mathcal{A}_{r} \mathcal{X}_{r}=\mathcal{B}_{r}$ then we get a prediction

$$
q_{k}^{(0)}(s, r+1)=E_{k}\left(s, r, \mathcal{X}_{r}^{(0)}\right), k=1,2,3, s=0, \ldots, s_{0}-r+1 .
$$

The first stage of the correction process is carried out by adding rows above $\mathcal{A}_{r}$ and $\mathcal{B}_{r}$, that is, we now obtain $\mathcal{X}_{r}^{(1)}$ from the over-determined system $\left[\mathcal{A}_{r}^{\prime} ; \mathcal{A}_{r}\right] \mathcal{X}_{r}=\left[\mathcal{B}_{r}^{\prime} ; \mathcal{B}_{r}\right]$. Finally we obtain a corrected value $q_{k}^{(1)}(s, r+1)$ from $(5.10)$ when $\mathcal{X}_{r}^{(1)}$ takes the place of $\mathcal{X}_{r}^{(0)}$. Our calculations have involved matrices $\mathcal{A}_{r}^{\prime}$ having up to 25 rows. An estimate $\mathcal{X}_{r}^{(1)}$ can also be obtained by solving $\mathcal{A}_{r}^{\prime} \mathcal{X}_{r}=\mathcal{B}_{r}^{\prime}$.

Table 5.5 suggests that the routines to simulate the rational simultaneous rule used in Test $2\left(\mathrm{SRQF}^{(2)}\right)$ are less stable than those used in Test $1\left(\mathrm{SRQF}^{(1)}\right)$. It can also be appraised that for $\operatorname{deg} \alpha \geq 2$ our approach seems to be superior to that presented in [9] (see column RGQF of Table 5.5).

6. Conclusions. Extensive testing confirms the theoretical geometrical rate of convergence of the SRQF, which can be reached with our numerical approach when the integrand is analytic in a neighborhood of the integration interval, even when some poles are close to the integration interval.

In principle, the technique of changing the variable is much more flexible than those ones based on rational quadrature formulas, and can always be applied directly to the integral of an analytic function with difficult real poles. Nevertheless, the more specific nature of rational rules makes them eventually more efficient. An argument in favor of combining both techniques smoothing transformation and rational rules, 
TABLE 5.5

Relative errors obtained in Test 2 when the integral $J_{1}(0.001)$ is evaluated by a simultaneous rational rule $\left(S R Q F^{(2)}\right)$, and by a rational Gaussian method ( $R G Q F$ )

\begin{tabular}{cccc|cccc}
\hline$r$ & $\operatorname{deg} \alpha$ & RGQF & SRQF $^{(2)}$ & $r$ & $\operatorname{deg} \alpha$ & RGQF & SRQF $^{(2)}$ \\
\hline 4 & 2 & $2.799 \mathrm{e}-05$ & $5.498 \mathrm{e}-07$ & 2 & 1 & $4.335 \mathrm{e}-03$ & $1.666 \mathrm{e}-02$ \\
6 & 3 & $5.383 \mathrm{e}-07$ & $2.153 \mathrm{e}-12$ & 3 & 1 & $1.210 \mathrm{e}-04$ & $5.396 \mathrm{e}-04$ \\
8 & 4 & $1.392 \mathrm{e}-08$ & $2.991 \mathrm{e}-13$ & 4 & 1 & $3.379 \mathrm{e}-06$ & $2.862 \mathrm{e}-05$ \\
10 & 5 & $9.281 \mathrm{e}-08$ & $1.508 \mathrm{e}-10$ & 5 & 1 & $9.281 \mathrm{e}-08$ & $1.496 \mathrm{e}-06$ \\
\hline 3 & 2 & $2.059 \mathrm{e}-03$ & $2.376 \mathrm{e}-05$ & 9 & 6 & $2.037 \mathrm{e}-06$ & $2.400 \mathrm{e}-09$ \\
6 & 4 & $7.991 \mathrm{e}-05$ & $4.067 \mathrm{e}-12$ & 12 & 8 & $1.392 \mathrm{e}-08$ & $3.369 \mathrm{e}-12$ \\
\hline
\end{tabular}

consists in that the former seems to be more effective in calculating the modified moments (5.3) than the integral under interest.

The enlargement of the basic system $\mathcal{A}_{r} \mathcal{X}_{r}=\mathcal{B}_{r}$ by adding rows from below is optional and must follow principle I, though its contribution in improving the accuracy in Test 1 has not been relevant. It seems that the technique of adding rows, possibly non consecutives, from below and/or above decreases the adverse effect produced by very small singular values of $\mathcal{A}_{r}$. In all cases the number of rows to be added must satisfy principle II.

All the computations have been performed using Matlab ${ }^{\circledR}$ tools.

\section{REFERENCES}

[1] N. I. ACHIEZER, Theory of approximation, UNGAR. New York, 1956.

[2] A. Branquinho, J. Bustamante, A. Folquié, and G. López Lagomasino, Normal indices in Nikishin systems, J. Approx. Theory, 124 (2003), 254-262.

[3] J. Bustamante and G. Lopez Lagomasino, Hermite-Padé Approximation for Nikishin systems of analytic functions. Russian Acad. Sci. Sb. Math. 77 (1994), 367-384.

[4] C. F. Borges, On a class of Gauss-like quadrature rules, Num. Math. 67 (1994), 271-288

[5] K. Driver and H. Stahl, Normality in Nikishin systems. Indag. Mathem., N.S., 5 (2)(1994), 161-187.

[6] U. Fidalgo and G. López lagomasino, On perfect Nikishin systems, Comp. Methods and Function Theory, 2 (2002), 415-426.

[7] _ Rate of convergence of generalized Hermite-Padé approximants of Nikishin systems, To appear in Constr. Approx.

[8] U. Fidalgo Prieto, J. R. Illán and G. López Lagomasino, Hermite-Padé approximation and simultaneous quadrature formulas, J. Approx. Theory, 126 (2004), 171-197.

[9] W. Gautschi, Algorithm 793: GQRAT-Gauss quadrature for rational functions, ACM Trans. Math. Software, 25, 2 (1999), pp. 213-239.

[10] - The use of rational functions in numerical quadrature, J. Comput. Appl. Math. 133 (2001), pp. 111-126.

[11] W. Gautschi, L. Gori, M.L. Lo CAscio Quadrature rules for rational functions, Numer. Math. 86 (2000), pp. 617-633.

[12] A.A. Gonchar, On the rate of rational approximation to continuous functions with characteristic singularities, Mat Sb., 73 (115) (1967), pp. 630-638; [Math. USSR Sb., 2 (1967), pp. 561-568]

[13] A. A. Gonchar and G. López Lagomasino, On Markov's theorem for multipoint Padé approximants, Mat. Sb., 105 (147) (1978), 512-524; translated to english in Math. USSR Sb., 34 (1978), 449-459.

[14] J. R. ILLÁN, A quadrature formula of rational type for integrands with one endpoint singularity, Electron. Trans. Numer. Anal. 16, 2003, pp. 143-164.

[15] G. López-Lagomasino and J. R. IlLÁn, Numerical integration based on interpolation and their connection with rational approximation, Cienc. Mat. (Havana), Vol. 8 No. 2 (1987), pp. 31-44 (in spanish).

[16] G. Min, Lagrange interpolation and quadrature formula in rational systems, J. Approx. Theory 95, (1988), pp. 123-145. 
[17] G. Monegato, Quadrature formulas for functions with poles near the interval of integration, Math. Comp. Vol. 47, No. 175, (1986), pp. 301-312.

[18] G. Monegato, And L. Scuderi, Numerical integration of functions with boundary singularities, J. Comput. Appl. Math. 112 (1999), pp. 201-214.

[19] E. M. Nikishin, On simultaneous Padé Approximants. Mat. Sb. Tom. 113 (155) (1980), No. 4 [Math. USSR Sb. 41 (1982), No. 4 409-425]

[20] G. SzËGO, Orthogonal Polynomials, American Mathematical Society, Providence, Rhode Island, 1939.

[21] I. H. Sloan and W. E. Smith, Properties of interpolatory product integration rules, SIAM J. Numer. Anal. Vol. 19, No. 2 (1982), 427-442.

[22] W. VAn Assche, AND I. VANheRWEgen, Quadrature formulas based on rational interpolation, Math. Comput. 61, (204) (1993), pp. 765-783.

[23] J. L. WALSH, Interpolation and approximation by rational functions in the complex domain, American Mathematical Society, Providence, Rhode Island, 1969. 